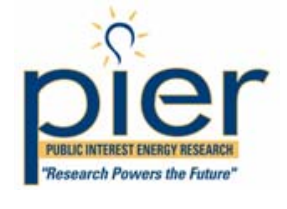

\title{
PIER Lighting Research Program
}

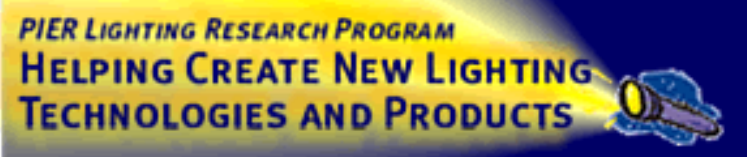

PIER Lighting Restarch Program

Helping Create New Lighting

TECHNOLOGIES AND PRODUCTS

California Energy Commission

Contract \# 500-01-041

\section{Draft Photosensor Characterization Report \\ Deliverable 3.3.1c}

February 28, 2003

Submitted To:

Accounting Office, MS-2

California Energy Commission

1516 Ninth Street, $1^{\text {st }}$ Floor

Sacramento, CA 95814

Submitted By:

Architectural Energy Corporation 2540 Frontier Avenue, Suite 201

Boulder, Colorado 80301 


\section{Table of Contents}

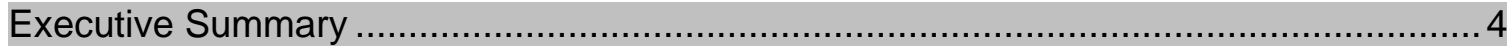

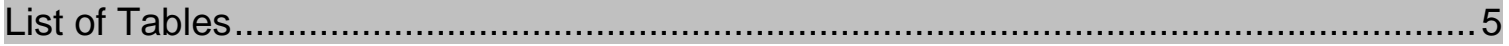

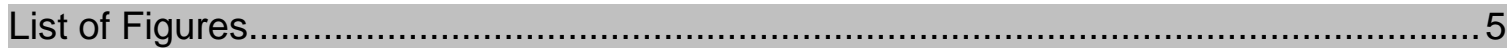

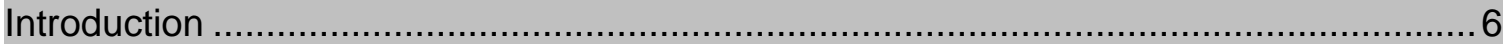

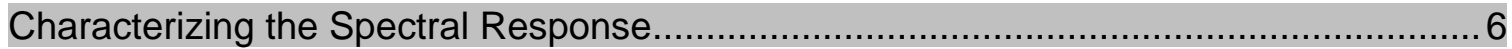

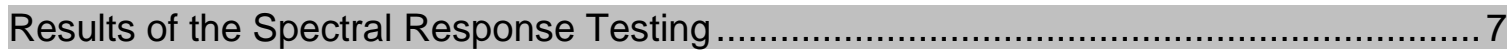

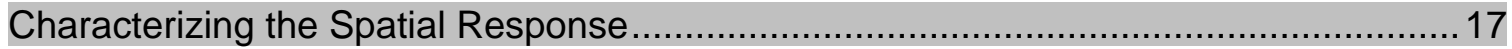

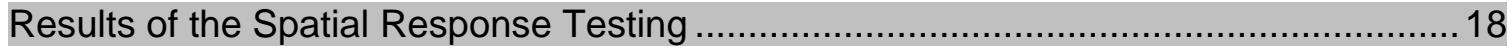

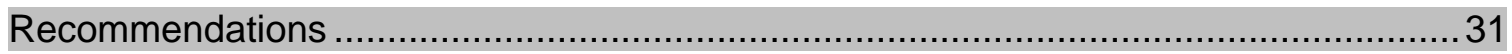

Contact Information:

Subcontract Project Manager

Doug Paton

The Watt Stopper

2800 De La Cruz Blvd.

Santa Clara, CA 95050

(925)-454-8224 voice

(925)-243-8912 fax

Doug Paton@WattStopper.com
AEC Program Director

Judie Porter

Architectural Energy Corporation

2540 Frontier Avenue

Boulder, CO 80301

303-444-4149 - Voice

303-444-4304 - Fax

jporter@archenergy.com

Prepared By:

Francis Rubinstein, Building Technologies Department, Lawrence Berkeley National Laboratory Mehry Yazdanian, Building Technologies Department, Lawrence Berkeley National Laboratory Jim Galvin, Building Technologies Department, Lawrence Berkeley National Laboratory

Project Team

Dr. Richard G. Mistrick, Associate Professor of Architectural Engineering, Pennsylvania State University

Dorene Maniccia, Manager, Market Segment Development, The Watt Stopper 
This work was completed under contract to Lawrence Berkeley National Laboratory as part of the California Energy Commission's Lighting Research Program. This program is supported by the California Energy Commission's Public Interest Energy Research (PIER) Buildings Program and the Assistant Secretary for Energy Efficiency and Renewable Energy, Office of Building Technology, Building Technologies Program, of the U.S. Department of Energy under Contract No. DE-AC03-76SF00098.

THIS REPORT WAS PREPARED AS A RESULT OF WORK SPONSORED BY THE CALIFORNIA ENERGY COMMISSION (COMMISSION). IT DOES NOT NECESSARILY REPRESENT THE VIEWS OF THE COMMISSION, ITS EMPLOYEES, OR THE STATE OF CALIFORNIA. THE COMMISSION, THE STATE OF CALIFORNIA, ITS EMPLOYEES, CONTRACTORS, AND SUBCONTRACTORS MAKE NO WARRANTY, EXPRESS OR IMPLIED, AND ASSUME NO LEGAL LIABILITY FOR THE INFORMATION IN THIS REPORT; NOR DOES ANY PARTY REPRESENT THAT THE USE OF THIS INFORMATION WILL NOT INFRINGE UPON PRIVATELY OWNED RIGHTS. THIS REPORT HAS NOT BEEN APPROVED OR DISAPPROVED BY THE COMMISSION NOR HAS THE COMMISSION PASSED UPON THE ACCURACY OR ADEQUACY OF THE INFORMATION IN THIS REPORT.

(C)2003, THE WATT STOPPER ALL RIGHTS RESERVED. 


\section{EXECUTIVE SUMMARY}

The report presents the results of laboratory measurements performed on The Watt Stopper's LS-201 photosensor at the Lawrence Berkeley National Laboratory in January 2003. The purpose of these measurements was to characterize the spatial and spectral response function of the LS-201 photosensor. Sample results of the spectral response and spatial response are shown below.
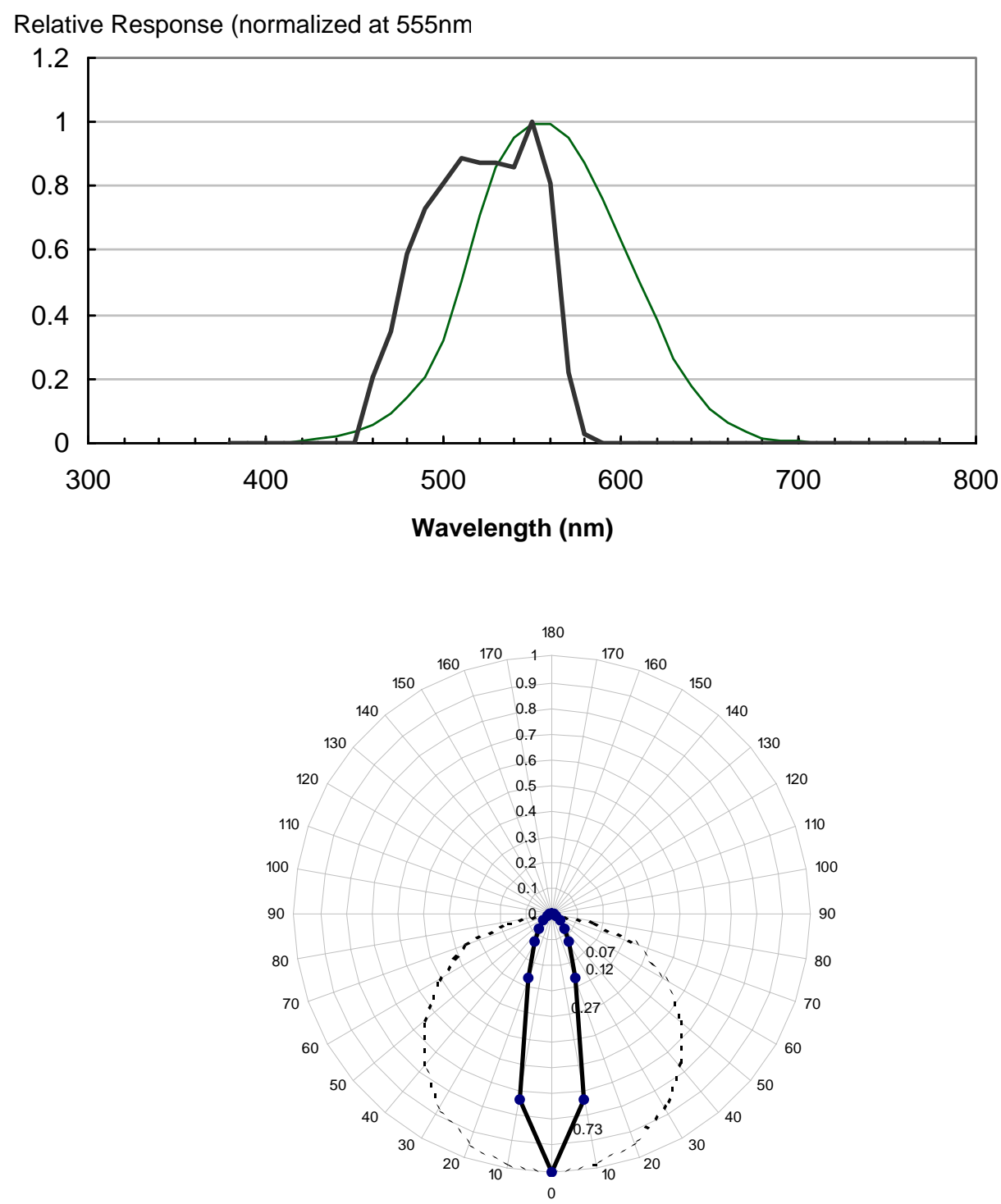


\section{List of Tables}

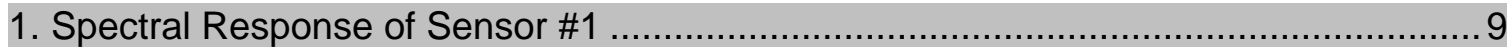

2. Spatial Response of Sensor \#1 with Lens ………............................................. 18

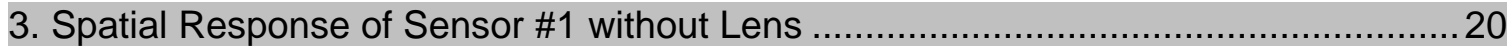

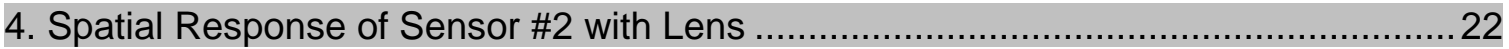

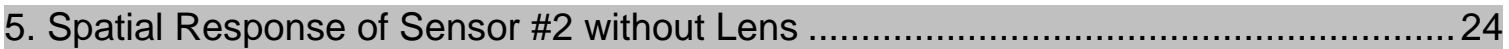

\section{List of Figures}

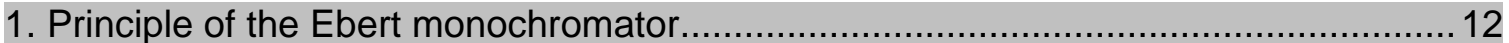

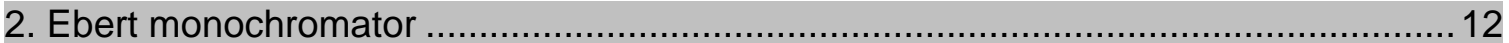

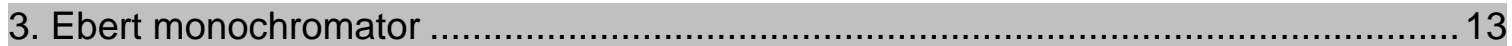

4. Monochromator sample and light source position................................................ 13

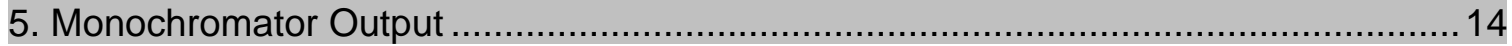

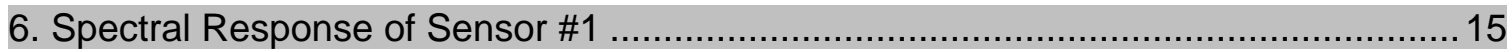

7. Effective Transmittances of Lens of Sensor \#1 ....................................................... 15

8. Spectral Response of Photocell Compared to Photopic Curve................................... 16

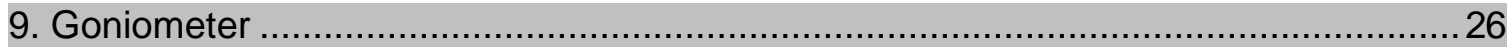

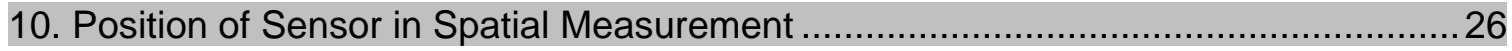

11. Sensor and Light Source Position in Spatial Measurements …............................. 27

12. Spatial Response of Sensor \#1 with Lens ………...................................... 28

13. Spatial Response of Sensor \#1 without Lens ……............................................. 28

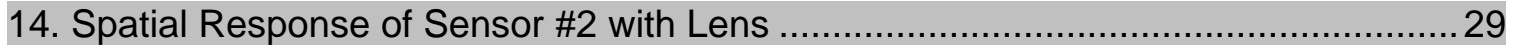

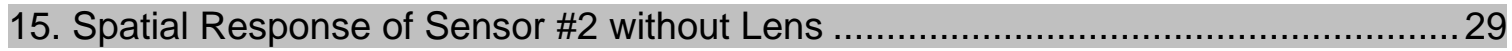

16. Polar Plot of Spatial Responsivity of Sensor \#1 ……............................................ 30 


\section{Introduction}

This report presents the results of laboratory measurements performed on The Watt Stopper's LS-201 photosensor at the Lawrence Berkeley National Laboratory in January, 2003. The purpose of these measurements was to characterize the spatial and spectral response function of the LS-201 photosensor. This work was performed in fulfillment of a Project 3.3 Deliverable for the California Energy Commission's Lighting Research Program.

The report has three sections. The first section describes the measurements performed to determine the spectral response function (sensitivity to light of different wavelengths) of the photosensor. The second section presents the results of our measurements of the sensitivity of the photosensor to light from different directions - the spatial response function. The third section presents our conclusions and preliminary suggestions for product improvement consistent with project goals and objectives

\section{Characterizing the Spectral Response}

The performance of any control photosensor depends on many variables, including its ability to measure the spectral composition of the light within the space in a similar manner as the human eye. The spectral response of a photosensor is expressed as photosensor sensitivity as a function of wavelength of the incident radiation. Previous work has shown that large mismatches between the spectral response of the photosensor and the human eye (as given by the photopic function) can cause significant errors in daylighting control systems. Once the spectral response function of a photosensor has been measured, it is straightforward to compute potential errors in system response solely from knowledge of the spectral composition of the light in the space (fluorescent, daylight, etc).

In Project 3.3, the LBNL Lighting Lab is assisting The Watt Stopper by measuring the spectral response function of candidate photosensors. Since the LS-201 photosensor is the point of departure for subsequent improved designs, we measured the spectral response of this photocell. The purpose of these measurements were to characterize the spectral performance of the photosensor by measuring the spectral response of the photosensor to incident light.

\section{Method}

The spectral response of the photosensor was measured by exposing the photosensor to known amounts of monochromic radiation, and measuring the resulting photocell voltage. We used an Ebert monochromator (model JA 82-000), equipped with a 1200 groove per mm grating, to produce quasi-monochromatic radiation from a broadband light source. Figures 1 and 2 show the theory and the design of the Ebert monochromator. Light from a microscope illuminator was focused onto an input $1 \mathrm{~mm}$ slit (Figures 3 and 
4). The light passing through this slit, was collimated by a concave mirror, which also reflected it onto a diffraction grating. The grating, in turn, directed the light onto a second concave mirror, which reflected and focused it onto an exit slit. Mirrors were used rather than lenses because they do not introduce any chromatic aberration. Because the redirection of the light beam by the grating is actually a diffraction rather than an ordinary reflection, the grating disperses the beam, i.e. different wavelengths leave the grating at different angles. By manually rotating the grating about its central axis, we varied the range of wavelengths, which reflected and focused by the second mirror onto the exit slit. The sensor was placed in front of the exit slit (Figure 4). The output of the photosensor was connected to a voltmeter. The maximum output range for our instrument was from 370 to 750 nanometers. However, due to low system throughput and low sensitivity of the photosensor, the practical range for the sensors we measured was from 440 to 610 nanometers, starting at $610 \mathrm{~nm}$ and going down to 440nm. Beyond this range, we did not notice any change in the readings.

We measured the spectral response of two LS201, with and without the lens. These photosensors had been modified by The Watt Stopper for the test so that the photosensors acted like meters (rather than as active control photosensors which would make accurate readings of the response impossible). To measure the actual throughput of the monochrometer, we replaced the photosensor with a silicon-based irradiance probe (Tektronix J6502A) with a known flat response function over the visible range. For this measurement, we replaced the sensor with the photometer. All measurements were taken at 10 -nm increments. For all the measurements, we placed the sensor as close as possible to the exit slit, covered the sensor, and turned off the overhead room lights.

\section{Results of the Spectral Response Testing}

For this report, we only included the results for one sensor because the test set-up for the second sensor was inadvertently jarred during measurement resulting in artifact that could not be corrected without redoing the measurement. The tabulated data results are summarized in Table 1 and key spectral characteristics given in Figures 5, 6, and 7. Table 1 lists the results of the sensor response, with and without lens, and the output of the monochromator. We corrected the sensor measurements for the offset by subtracting the sensor measurement at the starting point $(640 \mathrm{~nm})$ from each data point. We then calculated the normalized response by dividing the sensor response by the output of the monochromator. We also calculated the effective transmittances of the lens by dividing the normalized response with lens by the normalized response without lens. Figures 6 and 7 show the results of our measurements. Note that this is not the actual spectral sensitivity of the photosensor as it is not corrected for non-zero offset or the variable throughput of the monochrometer. Figure 6 gives the spectral response function for the Sensor \#1 (with and without lens) corrected for offset and monochrometer throughput. Figure 7 shows the approximate transmittance of the lens alone. It is reasonably flat, as would be expected for a lens exhibiting no obvious color. 
Figure 8 superimposes the spectral responsivity of Sensor \#1 with the photopic function (both curves normalized to unity at $555 \mathrm{~nm}$ ). From the Figure 8, it is seen that the spectral response of Sensor \#1 is similar in shape to the photopic curve but slightly narrower (80 $\mathrm{nm}$ fullwidth at half maximum compared to $100 \mathrm{~nm}$ FWHM for the photopic curve). Note also that the weighted center wavelength of the Sensor \#1 is translated about $40 \mathrm{~nm}$ toward the blue.

To calculate the error due to the mismatch in spectral responses of the TWS sensor and a perfectly color corrected sensor (obeying the photopic curve), we use:

$$
a(Z)=\frac{s_{T}(Z)}{s_{T}(N)}
$$

where $\mathrm{a}(\mathrm{Z})$ is the ratio of the responsivity $\mathrm{s}_{\mathrm{T}}(\mathrm{Z})$ when the test sensor is irradiated with radiation $\mathrm{Z}$ to the responsivity $\mathrm{s}_{\mathrm{T}}(\mathrm{N})$ when it is irradiated with a reference radiation $\mathrm{N}$. For control photosensors that work with daylight and modern fluorescent lamps, we suggest that the reference radiation $\mathrm{N}$ be the relative spectral response curve of a $3500 \mathrm{~K}$ fluorescent lamp (RE-735) and the test radiation $\mathrm{Z}$ be the relative spectral response of a phase of daylight (CIE Illuminant $\mathrm{D}_{65}$ ). The error in measuring the test radiation $\mathrm{D}_{65}$ is:

$$
\begin{aligned}
& f_{1}\left(D_{65}\right)=a\left(D_{65}\right)-1 \\
& f_{1}\left(D_{65}\right)=\left(s_{D} / s_{35}\right)-1
\end{aligned}
$$

Expanding we get:

$$
f_{1}(D)=\frac{\sum_{i=\min }^{\max } S\left(\lambda_{i}\right)_{D 65} V\left(\lambda_{i}\right)_{\text {rel }} \Delta \lambda}{\sum_{i=\min }^{\max } S\left(\lambda_{i}\right)_{D 65} r_{T}\left(\lambda_{i}\right)_{\text {rel }} \Delta \lambda} \bullet \frac{\sum_{i=\min }^{\max } S\left(\lambda_{i}\right)_{S P 35} r_{T}\left(\lambda_{i}\right)_{\text {rel }} \Delta \lambda}{\sum_{i=\min }^{\max } S\left(\lambda_{i}\right)_{S P 35} V\left(\lambda_{i}\right)_{r e l} \Delta \lambda}-1
$$

where $S\left(\lambda_{i}\right)_{D 65}$ is the relative spectral distribution curve for the CIE Illuminant D65, $r_{T}\left(\lambda_{i}\right)_{\text {rel }}$ is the relative spectral response of the test sensor, $V\left(\lambda_{i}\right)_{\text {rel }}$ is the relative photopic curve and $S\left(\lambda_{i}\right)_{S P 35}$ is the relative spectral distribution curve for a RE-35 fluorescent lamp.

As we did not have on a hand the SPD for a RE-35 lamp, we used the SPD from a RE-41 lamp that we did have on electronic file. [We will redo the calculation once we obtain the SPD for the RE-35]. Using the SP-41 as the reference, we calculated that the TWS sensor will err on the high side by about $15 \%$ when measuring daylight.

[1] Commission Internationale De L'Eclairage, "Methods of Characterizing the Performance of Radiometers and Photometers,” Publication CIE No. 53 (TC-2.2), 1982. 
Table 1-Spectral Response of Sensor \#1

\begin{tabular}{|c|c|c|c|c|c|c|c|c|}
\hline $\begin{array}{c}\text { Wavelength } \\
(\mathrm{nm})\end{array}$ & $\begin{array}{l}\text { With } \\
\text { Lens }\end{array}$ & $\begin{array}{l}\text { With } \\
\text { Lens } \\
\text { Corrected } \\
\text { for Zero } \\
\end{array}$ & $\begin{array}{l}\text { Without } \\
\text { Lens }\end{array}$ & $\begin{array}{l}\begin{array}{l}\text { Without } \\
\text { Lens } \\
\text { Corrected } \\
\text { for Zero }\end{array} \\
\end{array}$ & $\begin{array}{l}\text { Output of the } \\
\text { Monochromator }\end{array}$ & $\begin{array}{l}\text { With Lens } \\
\text { Normalized }\end{array}$ & $\begin{array}{l}\text { Without } \\
\text { Lens } \\
\text { Normalized }\end{array}$ & $\begin{array}{c}\text { Effective } \\
\text { Transmittances } \\
\text { of Lens }\end{array}$ \\
\hline 610 & 0.016 & 0.000 & 0.018 & 0.000 & 0.584 & 0.000 & 0.000 & 0.000 \\
\hline 609 & 0.017 & 0.000 & 0.018 & 0.000 & 0.577 & 0.000 & 0.000 & 0.000 \\
\hline 608 & 0.017 & 0.000 & 0.018 & 0.000 & 0.571 & 0.000 & 0.000 & 0.000 \\
\hline 607 & 0.017 & 0.000 & 0.018 & 0.000 & 0.564 & 0.000 & 0.000 & 0.000 \\
\hline 606 & 0.017 & 0.000 & 0.018 & 0.000 & 0.558 & 0.000 & 0.000 & 0.000 \\
\hline 605 & 0.017 & 0.000 & 0.018 & 0.000 & 0.551 & 0.000 & 0.000 & 0.000 \\
\hline 604 & 0.017 & 0.000 & 0.018 & 0.000 & 0.545 & 0.000 & 0.000 & 0.000 \\
\hline 603 & 0.017 & 0.000 & 0.018 & 0.000 & 0.540 & 0.000 & 0.000 & 0.000 \\
\hline 602 & 0.017 & 0.000 & 0.018 & 0.000 & 0.534 & 0.000 & 0.000 & 0.000 \\
\hline 601 & 0.017 & 0.000 & 0.018 & 0.000 & 0.529 & 0.000 & 0.000 & 0.000 \\
\hline 600 & 0.017 & 0.000 & 0.018 & 0.000 & 0.523 & 0.000 & 0.000 & 0.000 \\
\hline 599 & 0.017 & 0.000 & 0.018 & 0.000 & 0.522 & 0.000 & 0.000 & 0.000 \\
\hline 598 & 0.017 & 0.000 & 0.018 & 0.000 & 0.522 & 0.000 & 0.000 & 0.000 \\
\hline 597 & 0.017 & 0.000 & 0.018 & 0.000 & 0.521 & 0.000 & 0.000 & 0.000 \\
\hline 596 & 0.017 & 0.000 & 0.018 & 0.000 & 0.521 & 0.000 & 0.000 & 0.000 \\
\hline 595 & 0.017 & 0.000 & 0.019 & 0.001 & 0.520 & 0.000 & 0.002 & 0.000 \\
\hline 594 & 0.017 & 0.000 & 0.019 & 0.001 & 0.509 & 0.000 & 0.002 & 0.000 \\
\hline 593 & 0.017 & 0.000 & 0.019 & 0.001 & 0.497 & 0.000 & 0.002 & 0.000 \\
\hline 592 & 0.017 & 0.000 & 0.020 & 0.002 & 0.486 & 0.000 & 0.004 & 0.000 \\
\hline 591 & 0.017 & 0.000 & 0.020 & 0.002 & 0.474 & 0.000 & 0.004 & 0.000 \\
\hline 590 & 0.017 & 0.000 & 0.021 & 0.003 & 0.463 & 0.000 & 0.006 & 0.000 \\
\hline 589 & 0.018 & 0.001 & 0.021 & 0.003 & 0.456 & 0.002 & 0.007 & 3.000 \\
\hline 588 & 0.018 & 0.001 & 0.022 & 0.004 & 0.450 & 0.002 & 0.009 & 4.000 \\
\hline 587 & 0.018 & 0.001 & 0.023 & 0.005 & 0.443 & 0.002 & 0.011 & 5.000 \\
\hline 586 & 0.018 & 0.001 & 0.024 & 0.006 & 0.437 & 0.002 & 0.014 & 6.000 \\
\hline 585 & 0.019 & 0.002 & 0.025 & 0.007 & 0.430 & 0.005 & 0.016 & 3.500 \\
\hline 584 & 0.019 & 0.002 & 0.026 & 0.008 & 0.423 & 0.005 & 0.019 & 4.000 \\
\hline 583 & 0.020 & 0.003 & 0.028 & 0.010 & 0.416 & 0.007 & 0.024 & 3.333 \\
\hline 582 & 0.020 & 0.003 & 0.030 & 0.012 & 0.409 & 0.007 & 0.029 & 4.000 \\
\hline 581 & 0.021 & 0.004 & 0.032 & 0.014 & 0.402 & 0.010 & 0.035 & 3.500 \\
\hline 580 & 0.021 & 0.004 & 0.034 & 0.016 & 0.395 & 0.010 & 0.041 & 4.000 \\
\hline 579 & 0.022 & 0.005 & 0.037 & 0.019 & 0.388 & 0.013 & 0.049 & 3.800 \\
\hline 578 & 0.023 & 0.006 & 0.040 & 0.022 & 0.382 & 0.016 & 0.058 & 3.667 \\
\hline 577 & 0.024 & 0.007 & 0.044 & 0.026 & 0.375 & 0.019 & 0.069 & 3.714 \\
\hline 576 & 0.026 & 0.009 & 0.049 & 0.031 & 0.369 & 0.024 & 0.084 & 3.444 \\
\hline 575 & 0.027 & 0.010 & 0.057 & 0.039 & 0.362 & 0.028 & 0.108 & 3.900 \\
\hline 574 & 0.029 & 0.012 & 0.063 & 0.045 & 0.354 & 0.034 & 0.127 & 3.750 \\
\hline 573 & 0.032 & 0.015 & 0.072 & 0.054 & 0.346 & 0.043 & 0.156 & 3.600 \\
\hline 572 & 0.034 & 0.017 & 0.082 & 0.064 & 0.338 & 0.050 & 0.189 & 3.765 \\
\hline 571 & 0.038 & 0.021 & 0.095 & 0.077 & 0.330 & 0.064 & 0.233 & 3.667 \\
\hline 570 & 0.042 & 0.025 & 0.108 & 0.090 & 0.322 & 0.078 & 0.280 & 3.600 \\
\hline 569 & 0.046 & 0.029 & 0.121 & 0.103 & 0.315 & 0.092 & 0.327 & 3.552 \\
\hline 568 & 0.049 & 0.032 & 0.135 & 0.117 & 0.307 & 0.104 & 0.381 & 3.656 \\
\hline 567 & 0.054 & 0.037 & 0.138 & 0.120 & 0.300 & 0.123 & 0.400 & 3.243 \\
\hline 566 & 0.057 & 0.040 & 0.160 & 0.142 & 0.292 & 0.137 & 0.486 & 3.550 \\
\hline 565 & 0.060 & 0.043 & 0.171 & 0.153 & 0.285 & 0.151 & 0.537 & 3.558 \\
\hline 564 & 0.064 & 0.047 & 0.181 & 0.163 & 0.278 & 0.169 & 0.587 & 3.468 \\
\hline 563 & 0.070 & 0.053 & 0.203 & 0.185 & 0.270 & 0.196 & 0.685 & 3.491 \\
\hline 562 & 0.075 & 0.058 & 0.227 & 0.209 & 0.263 & 0.221 & 0.795 & 3.603 \\
\hline 561 & 0.081 & 0.064 & 0.247 & 0.229 & 0.255 & 0.251 & 0.897 & 3.578 \\
\hline 560 & 0.087 & 0.070 & 0.265 & 0.247 & 0.248 & 0.282 & 0.996 & 3.529 \\
\hline 559 & 0.092 & 0.075 & 0.281 & 0.263 & 0.241 & 0.311 & 1.092 & 3.507 \\
\hline 558 & 0.096 & 0.079 & 0.294 & 0.276 & 0.234 & 0.338 & 1.182 & 3.494 \\
\hline
\end{tabular}




\begin{tabular}{|c|c|c|c|c|c|c|c|c|}
\hline $\begin{array}{l}\text { Wavelength } \\
(\mathrm{nm})\end{array}$ & $\begin{array}{l}\text { With } \\
\text { Lens }\end{array}$ & $\begin{array}{l}\text { With } \\
\text { Lens } \\
\text { Corrected } \\
\text { for Zero }\end{array}$ & $\begin{array}{l}\text { Without } \\
\text { Lens }\end{array}$ & $\begin{array}{l}\text { Without } \\
\text { Lens } \\
\text { Corrected } \\
\text { for Zero }\end{array}$ & $\begin{array}{l}\text { Output of the } \\
\text { Monochromator }\end{array}$ & $\begin{array}{l}\text { With Lens } \\
\text { Normalized }\end{array}$ & $\begin{array}{l}\text { Without } \\
\text { Lens } \\
\text { Normalized }\end{array}$ & $\begin{array}{c}\text { Effective } \\
\text { Transmittances } \\
\text { of Lens }\end{array}$ \\
\hline 557 & 0.100 & 0.083 & 0.303 & 0.285 & 0.226 & 0.367 & 1.259 & 3.434 \\
\hline 556 & 0.102 & 0.085 & 0.309 & 0.291 & 0.219 & 0.388 & 1.328 & 3.424 \\
\hline 555 & 0.103 & 0.086 & 0.307 & 0.289 & 0.212 & 0.406 & 1.363 & 3.360 \\
\hline 554 & 0.101 & 0.084 & 0.300 & 0.282 & 0.206 & 0.408 & 1.370 & 3.357 \\
\hline 553 & 0.096 & 0.079 & 0.278 & 0.260 & 0.200 & 0.396 & 1.303 & 3.291 \\
\hline 552 & 0.089 & 0.072 & 0.257 & 0.239 & 0.193 & 0.372 & 1.236 & 3.319 \\
\hline 551 & 0.083 & 0.066 & 0.234 & 0.216 & 0.187 & 0.353 & 1.154 & 3.273 \\
\hline 550 & 0.080 & 0.063 & 0.222 & 0.204 & 0.181 & 0.348 & 1.127 & 3.238 \\
\hline 549 & 0.076 & 0.059 & 0.209 & 0.191 & 0.176 & 0.336 & 1.086 & 3.237 \\
\hline 548 & 0.072 & 0.055 & 0.199 & 0.181 & 0.171 & 0.322 & 1.061 & 3.291 \\
\hline 547 & 0.069 & 0.052 & 0.187 & 0.169 & 0.165 & 0.314 & 1.022 & 3.250 \\
\hline 546 & 0.066 & 0.049 & 0.177 & 0.159 & 0.160 & 0.306 & 0.993 & 3.245 \\
\hline 545 & 0.064 & 0.047 & 0.168 & 0.150 & 0.155 & 0.303 & 0.968 & 3.191 \\
\hline 544 & 0.062 & 0.045 & 0.161 & 0.143 & 0.151 & 0.298 & 0.948 & 3.178 \\
\hline 543 & 0.060 & 0.043 & 0.154 & 0.136 & 0.147 & 0.293 & 0.928 & 3.163 \\
\hline 542 & 0.059 & 0.042 & 0.150 & 0.132 & 0.142 & 0.295 & 0.927 & 3.143 \\
\hline 541 & 0.058 & 0.041 & 0.144 & 0.126 & 0.138 & 0.297 & 0.912 & 3.073 \\
\hline 540 & 0.057 & 0.040 & 0.140 & 0.122 & 0.134 & 0.299 & 0.910 & 3.050 \\
\hline 539 & 0.056 & 0.039 & 0.136 & 0.118 & 0.131 & 0.298 & 0.902 & 3.026 \\
\hline 538 & 0.055 & 0.038 & 0.132 & 0.114 & 0.128 & 0.298 & 0.893 & 3.000 \\
\hline 537 & 0.054 & 0.037 & 0.129 & 0.111 & 0.124 & 0.297 & 0.892 & 3.000 \\
\hline 536 & 0.053 & 0.036 & 0.125 & 0.107 & 0.121 & 0.297 & 0.883 & 2.972 \\
\hline 535 & 0.053 & 0.036 & 0.122 & 0.104 & 0.118 & 0.305 & 0.881 & 2.889 \\
\hline 534 & 0.052 & 0.035 & 0.119 & 0.101 & 0.115 & 0.303 & 0.875 & 2.886 \\
\hline 533 & 0.051 & 0.034 & 0.116 & 0.098 & 0.113 & 0.301 & 0.869 & 2.882 \\
\hline 532 & 0.051 & 0.034 & 0.114 & 0.096 & 0.110 & 0.309 & 0.871 & 2.824 \\
\hline 531 & 0.050 & 0.033 & 0.112 & 0.094 & 0.108 & 0.307 & 0.874 & 2.848 \\
\hline 530 & 0.049 & 0.032 & 0.109 & 0.091 & 0.105 & 0.305 & 0.867 & 2.844 \\
\hline 529 & 0.049 & 0.032 & 0.108 & 0.090 & 0.103 & 0.310 & 0.872 & 2.813 \\
\hline 528 & 0.048 & 0.031 & 0.106 & 0.088 & 0.101 & 0.306 & 0.868 & 2.839 \\
\hline 527 & 0.048 & 0.031 & 0.104 & 0.086 & 0.100 & 0.311 & 0.863 & 2.774 \\
\hline 526 & 0.047 & 0.030 & 0.102 & 0.084 & 0.098 & 0.307 & 0.859 & 2.800 \\
\hline 525 & 0.047 & 0.030 & 0.101 & 0.083 & 0.096 & 0.313 & 0.865 & 2.767 \\
\hline 524 & 0.046 & 0.029 & 0.100 & 0.082 & 0.095 & 0.307 & 0.867 & 2.828 \\
\hline 523 & 0.046 & 0.029 & 0.098 & 0.080 & 0.093 & 0.311 & 0.858 & 2.759 \\
\hline 522 & 0.045 & 0.028 & 0.097 & 0.079 & 0.092 & 0.305 & 0.861 & 2.821 \\
\hline 521 & 0.045 & 0.028 & 0.095 & 0.077 & 0.090 & 0.310 & 0.852 & 2.750 \\
\hline 520 & 0.044 & 0.027 & 0.094 & 0.076 & 0.089 & 0.303 & 0.854 & 2.815 \\
\hline 519 & 0.044 & 0.027 & 0.094 & 0.076 & 0.087 & 0.310 & 0.872 & 2.815 \\
\hline 518 & 0.044 & 0.027 & 0.093 & 0.075 & 0.085 & 0.316 & 0.878 & 2.778 \\
\hline 517 & 0.043 & 0.026 & 0.091 & 0.073 & 0.084 & 0.311 & 0.873 & 2.808 \\
\hline 516 & 0.042 & 0.025 & 0.088 & 0.070 & 0.082 & 0.306 & 0.856 & 2.800 \\
\hline 515 & 0.042 & 0.025 & 0.085 & 0.067 & 0.080 & 0.313 & 0.838 & 2.680 \\
\hline 514 & 0.041 & 0.024 & 0.083 & 0.065 & 0.078 & 0.307 & 0.831 & 2.708 \\
\hline 513 & 0.040 & 0.023 & 0.082 & 0.064 & 0.076 & 0.301 & 0.838 & 2.783 \\
\hline 512 & 0.040 & 0.023 & 0.080 & 0.062 & 0.075 & 0.308 & 0.831 & 2.696 \\
\hline 511 & 0.039 & 0.022 & 0.078 & 0.060 & 0.073 & 0.302 & 0.824 & 2.727 \\
\hline 510 & 0.039 & 0.022 & 0.076 & 0.058 & 0.071 & 0.310 & 0.817 & 2.636 \\
\hline 509 & 0.038 & 0.021 & 0.074 & 0.056 & 0.070 & 0.302 & 0.805 & 2.667 \\
\hline 508 & 0.038 & 0.021 & 0.073 & 0.055 & 0.068 & 0.308 & 0.806 & 2.619 \\
\hline 507 & 0.037 & 0.020 & 0.071 & 0.053 & 0.067 & 0.299 & 0.793 & 2.650 \\
\hline 506 & 0.036 & 0.019 & 0.070 & 0.052 & 0.065 & 0.291 & 0.795 & 2.737 \\
\hline 505 & 0.036 & 0.019 & 0.067 & 0.049 & 0.064 & 0.297 & 0.766 & 2.579 \\
\hline 504 & 0.035 & 0.018 & 0.066 & 0.048 & 0.063 & 0.288 & 0.767 & 2.667 \\
\hline 503 & 0.035 & 0.018 & 0.065 & 0.047 & 0.061 & 0.294 & 0.768 & 2.611 \\
\hline 502 & 0.034 & 0.017 & 0.063 & 0.045 & 0.060 & 0.284 & 0.753 & 2.647 \\
\hline
\end{tabular}




\begin{tabular}{|c|c|c|c|c|c|c|c|c|}
\hline $\begin{array}{l}\text { Wavelength } \\
(\mathrm{nm})\end{array}$ & $\begin{array}{l}\text { With } \\
\text { Lens }\end{array}$ & $\begin{array}{c}\text { With } \\
\text { Lens } \\
\text { Corrected } \\
\text { for Zero } \\
\end{array}$ & $\begin{array}{l}\text { Without } \\
\text { Lens }\end{array}$ & $\begin{array}{c}\text { Without } \\
\text { Lens } \\
\text { Corrected } \\
\text { for Zero } \\
\end{array}$ & $\begin{array}{l}\text { Output of the } \\
\text { Monochromator }\end{array}$ & $\begin{array}{l}\text { With Lens } \\
\text { Normalized }\end{array}$ & $\begin{array}{c}\text { Without } \\
\text { Lens } \\
\text { Normalized }\end{array}$ & $\begin{array}{l}\text { Effective } \\
\text { Transmittances } \\
\text { of Lens }\end{array}$ \\
\hline 501 & 0.034 & 0.017 & 0.062 & 0.044 & 0.058 & 0.291 & 0.753 & 2.588 \\
\hline 500 & 0.033 & 0.016 & 0.061 & 0.043 & 0.057 & 0.281 & 0.754 & 2.688 \\
\hline 499 & 0.032 & 0.015 & 0.060 & 0.042 & 0.056 & 0.268 & 0.750 & 2.800 \\
\hline 498 & 0.033 & 0.016 & 0.059 & 0.041 & 0.055 & 0.291 & 0.745 & 2.563 \\
\hline 497 & 0.032 & 0.015 & 0.058 & 0.040 & 0.054 & 0.278 & 0.741 & 2.667 \\
\hline 496 & 0.032 & 0.015 & 0.056 & 0.038 & 0.053 & 0.283 & 0.717 & 2.533 \\
\hline 495 & 0.031 & 0.014 & 0.055 & 0.037 & 0.052 & 0.269 & 0.712 & 2.643 \\
\hline 494 & 0.031 & 0.014 & 0.054 & 0.036 & 0.051 & 0.275 & 0.706 & 2.571 \\
\hline 493 & 0.030 & 0.013 & 0.053 & 0.035 & 0.050 & 0.260 & 0.700 & 2.692 \\
\hline 492 & 0.030 & 0.013 & 0.052 & 0.034 & 0.049 & 0.265 & 0.694 & 2.615 \\
\hline 491 & 0.029 & 0.012 & 0.051 & 0.033 & 0.048 & 0.250 & 0.688 & 2.750 \\
\hline 490 & 0.029 & 0.012 & 0.049 & 0.031 & 0.047 & 0.255 & 0.660 & 2.583 \\
\hline 489 & 0.029 & 0.012 & 0.048 & 0.030 & 0.046 & 0.260 & 0.649 & 2.500 \\
\hline 488 & 0.028 & 0.011 & 0.047 & 0.029 & 0.045 & 0.242 & 0.639 & 2.636 \\
\hline 487 & 0.028 & 0.011 & 0.046 & 0.028 & 0.045 & 0.247 & 0.628 & 2.545 \\
\hline 486 & 0.027 & 0.010 & 0.045 & 0.027 & 0.044 & 0.228 & 0.616 & 2.700 \\
\hline 485 & 0.027 & 0.010 & 0.044 & 0.026 & 0.043 & 0.233 & 0.605 & 2.600 \\
\hline 484 & 0.026 & 0.009 & 0.043 & 0.025 & 0.042 & 0.213 & 0.592 & 2.778 \\
\hline 483 & 0.026 & 0.009 & 0.042 & 0.024 & 0.041 & 0.217 & 0.580 & 2.667 \\
\hline 482 & 0.026 & 0.009 & 0.040 & 0.022 & 0.041 & 0.222 & 0.542 & 2.444 \\
\hline 481 & 0.025 & 0.008 & 0.039 & 0.021 & 0.040 & 0.201 & 0.528 & 2.625 \\
\hline 480 & 0.025 & 0.008 & 0.038 & 0.020 & 0.039 & 0.205 & 0.513 & 2.500 \\
\hline 470 & 0.021 & 0.004 & 0.029 & 0.011 & 0.033 & 0.121 & 0.333 & 2.750 \\
\hline 460 & 0.019 & 0.002 & 0.023 & 0.005 & 0.028 & 0.071 & 0.179 & 2.500 \\
\hline 450 & 0.017 & 0.000 & 0.019 & 0.001 & 0.023 & 0.000 & 0.043 & 0.000 \\
\hline 440 & 0.017 & 0.000 & 0.018 & 0.000 & 0.019 & 0.000 & 0.000 & 0.000 \\
\hline
\end{tabular}




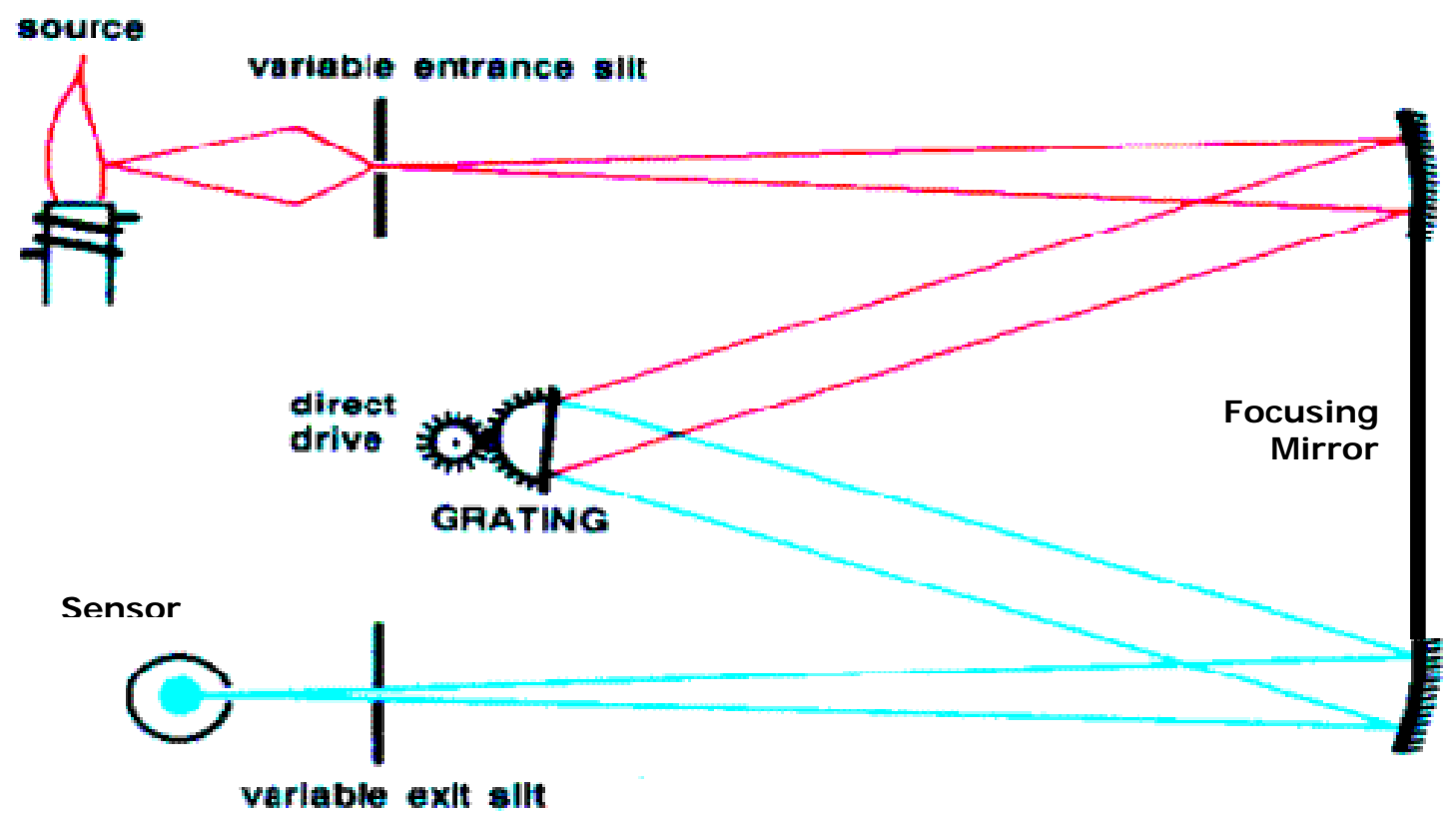

Figure 1-Principle of the Ebert monochromator

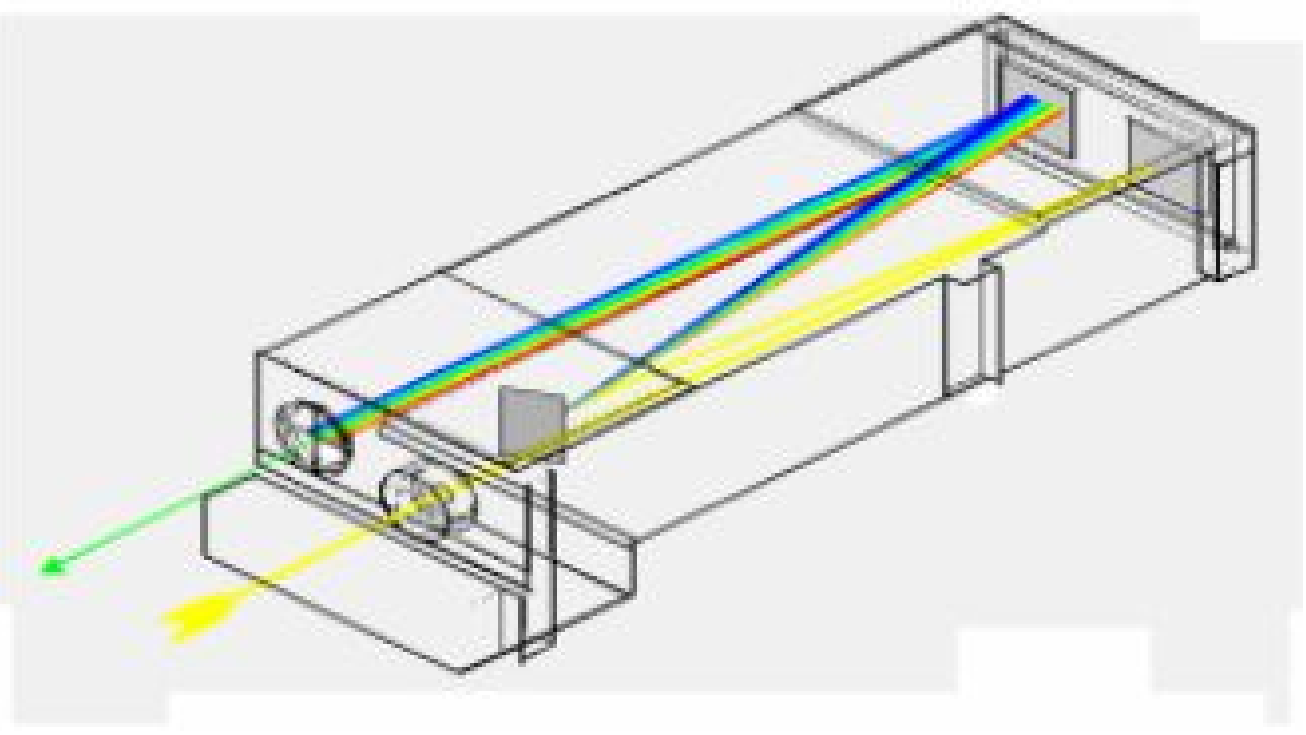

Figure 2-Ebert monochromator, consists of fixed entrance and exit slits, fixed focusing mirror and a rotatable diffraction grating. As the grating rotates a different wavelength is focused onto the exit slit. 


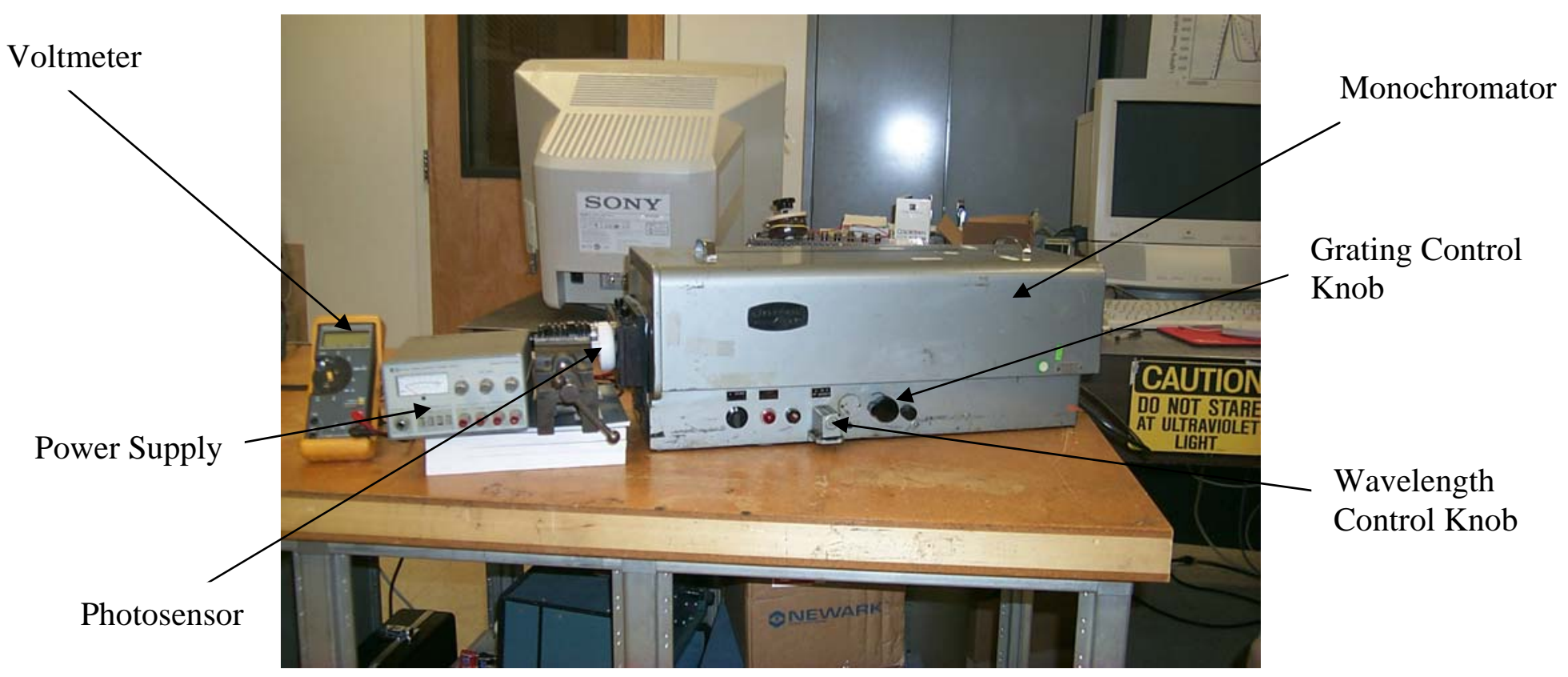

Figure 3-Ebert monochromator - model JA 82-000

Light Source (Microscope Illuminator)

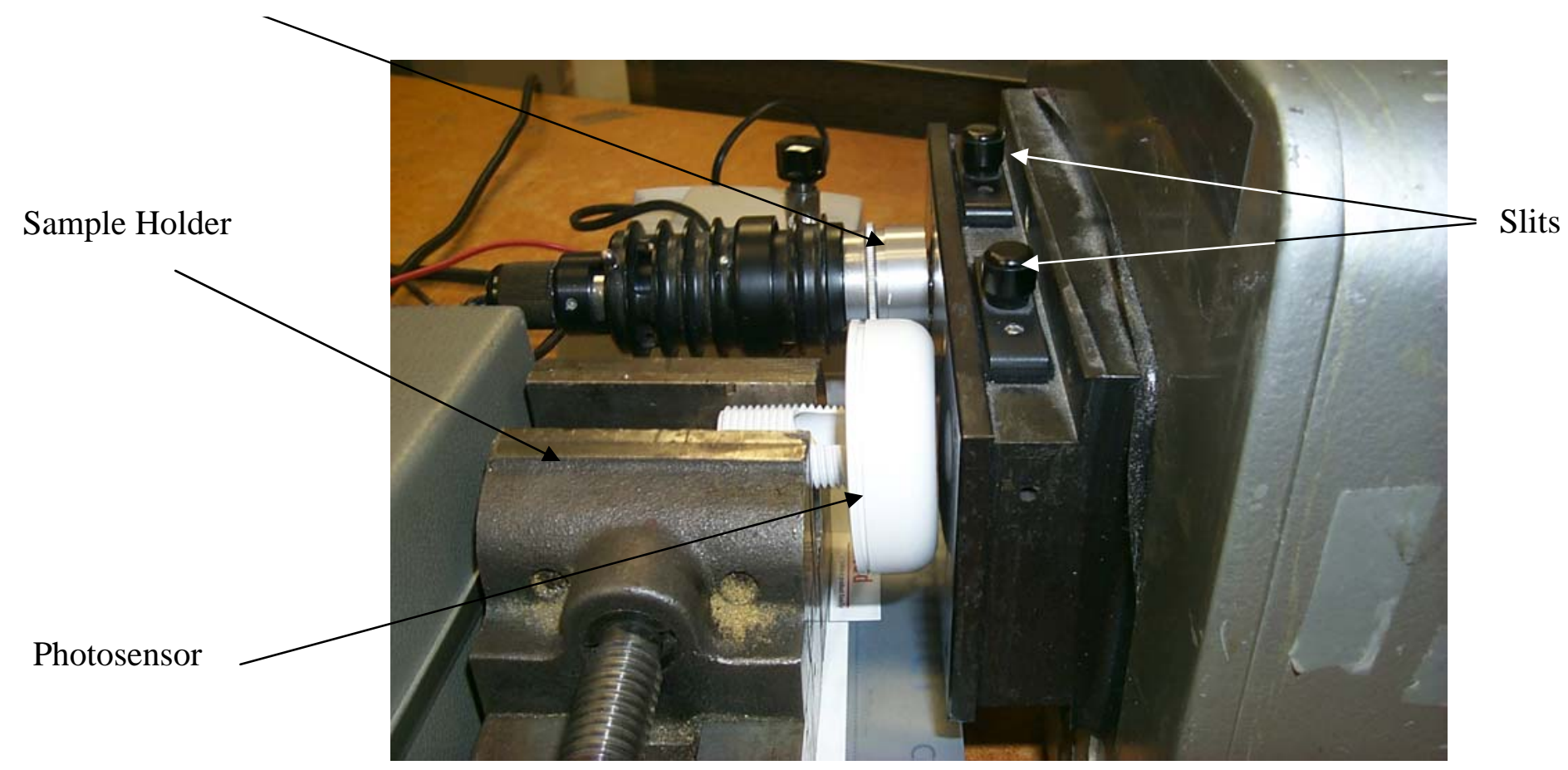

Figure 4-Monochromator Sample and Light source position 
Figure 5-Monochromator Output

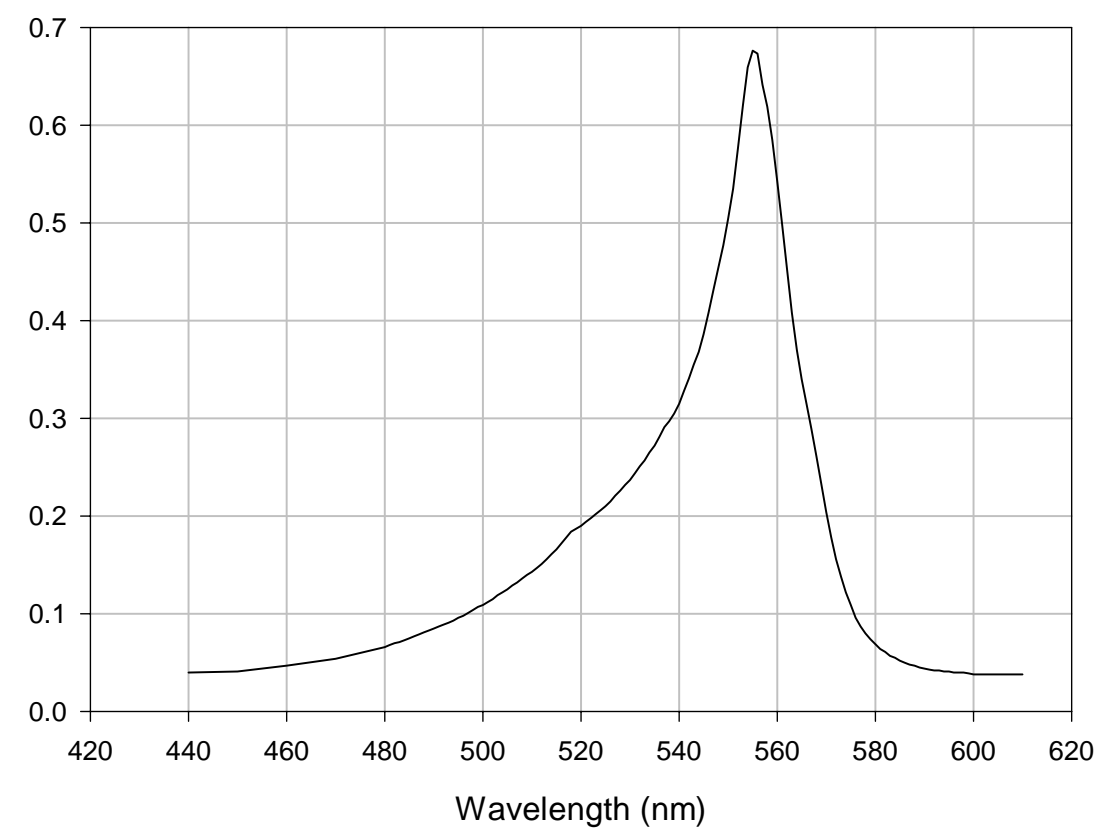


Figure 6-Spectral Response

Wattstoper Sensor\#1 Corrected for Zero \& Normalized

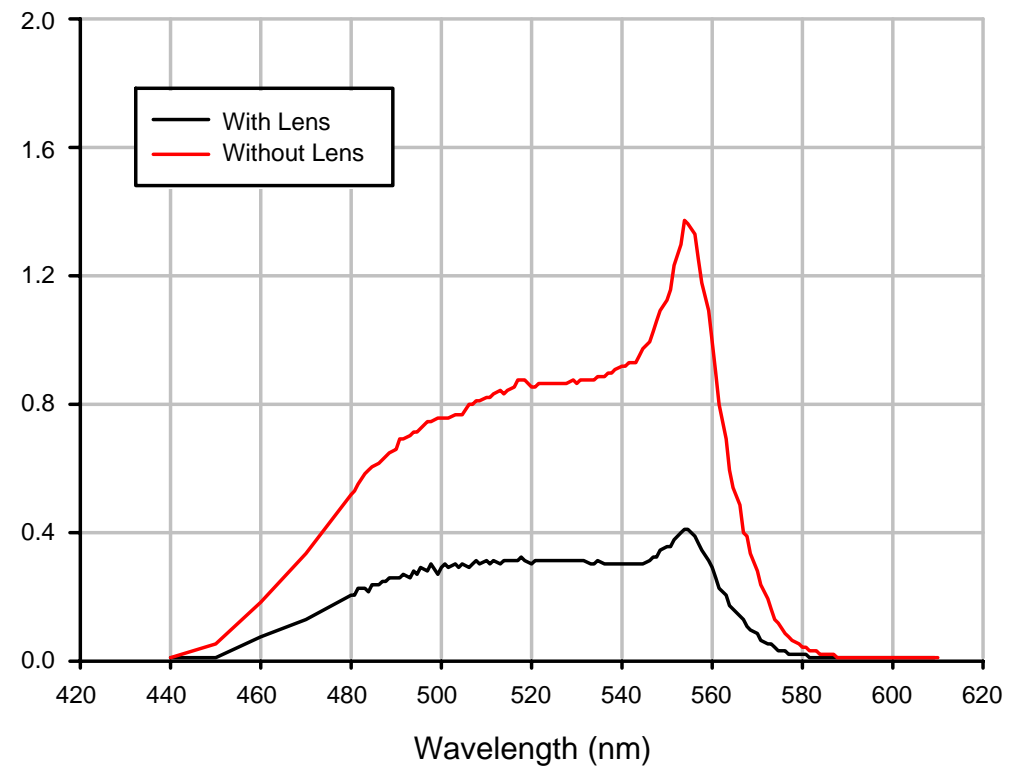

Figure 7-Sensor\#1Effective Transmittances of Lens

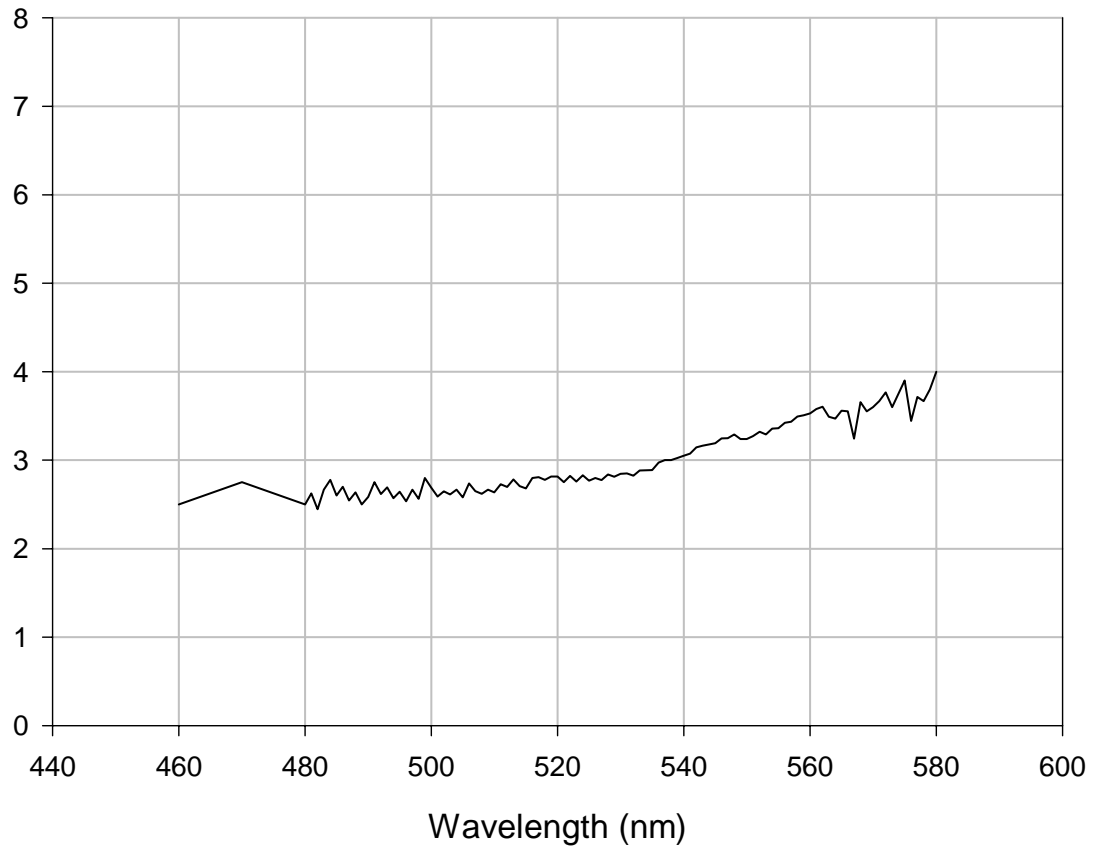


Figure 8

Spectral Response of Photocell Compared to Photopic Curve

Relative Response (normalized at 555nm

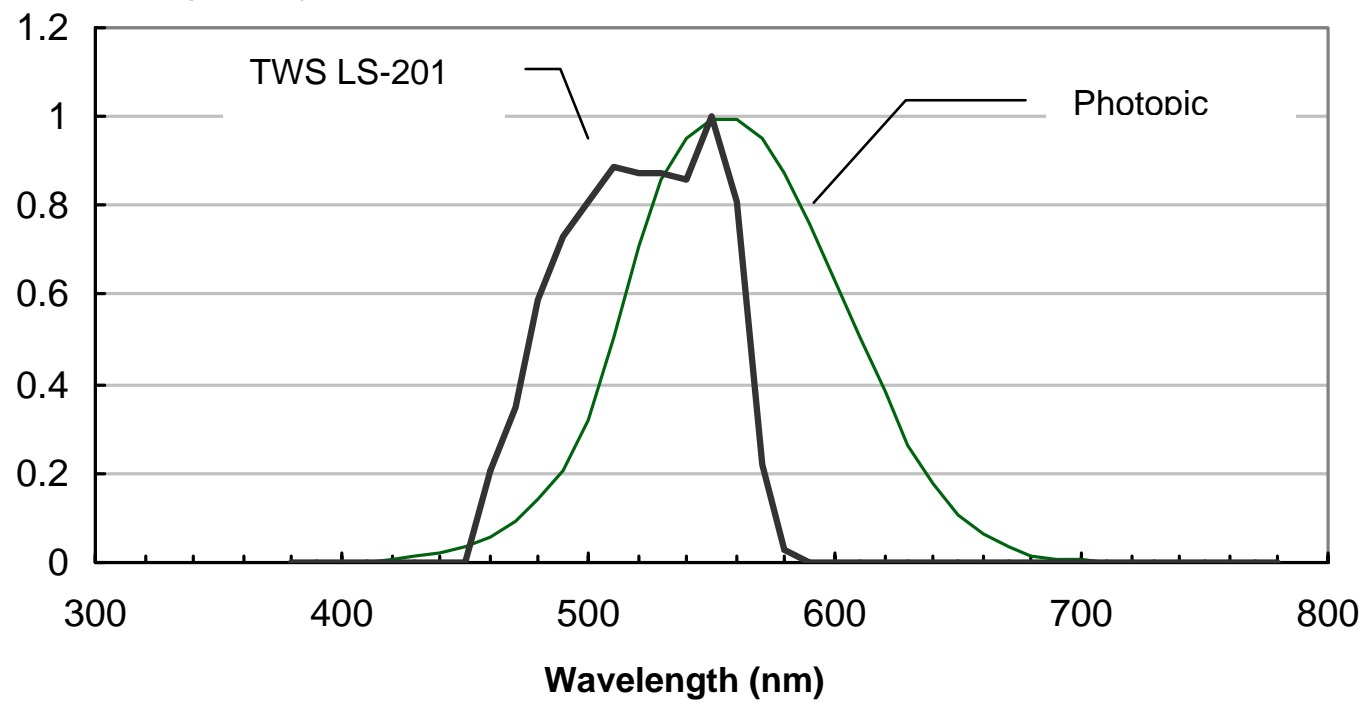




\section{Characterizing the Spatial Performance}

One factor affecting the performance of a control photosensor is its sensitivity to light striking it from different directions. The spatial response function (SRF) describes the sensitivity of the photosensor to incident radiation from coming from different directions within a space. Previous work has shown that SRFs differ from photosensor to photosensor. In some cases, the photocell viewed nearly an entire hemisphere with a roughly cosine response while others selectively sampled the space in different directions. It has also been shown the SRF and photosensor placement significantly affect overall control system performance particularly in cases where incorrect control algorithms are employed (viz. using integral reset for daylighting applications). In extreme cases, poorly designed systems can let light levels fall too far, causing occupant complaints and even sabotage.

In project 3.3, the LBNL Lighting Lab is assisting The Watt Stopper by measuring the spatial response function of candidate photosensors. The purpose of these measurements was to characterize the spatial performance of the photosensor by measuring the angular response of the photosensor to incident light.

\section{Method}

The spatial response of the photosensor was measured by affixing it at the photometric center of a miniature goniometer and subjecting the sensor to a light mounted to the movable arm. We used a two-axis goniometer (Figures 9 and 10) that was originally developed to measure the spatial distribution of light from novel light emitting diodes (LEDs). The goniophotometer, which consists of two perpendicular rotary stages which rotate around the vertical axis, measuring altitude, $\psi$, and the horizontal axis, measuring azimuth, $\theta$. The two angles, $\psi$ and $\theta$, defined the direction of light incident on the photosensor. We used the computer program Labview to program two stepper motors, through the serial port of the computer, to step the altitude angle, from -100 to 100 degrees, for all rotations of the azimuth angle. Measurements were taken every 10 degrees for both the altitude and azimuth axes. For each pair of altitude and azimuth angles $(\psi, \theta)$, we measured the response of the sensor. This method is exactly equivalent to the accepted method used to characterize the candlepower distribution from luminaries. The photosensor was mounted on the goniometer such that the center of the sensor was at the center of rotation. We used an aligning telescope to center the sensor. The distance between the sensor and the light source was $7 \mathrm{~cm}$ (Figure 10). The light source we used was a 3-Watt, clear incandescent 1487 pilot light. We used a regulated voltage source so that the light did not vary during the test. The output of the sensor (measured in volts) was read back into the computer, through a National Instrument data acquisition board (DAQ PCI-6034E), and was displayed on the Labview screen.

We measured the spatial response of two LS201, with and without lens. These photosensors had been modified by The Watt Stopper before the test so that the photosensors acted like meters (rather than as active control photosensors, which would 
have rendered accurate readings of the response impossible). For all the measurements, we covered the sensor and the light source with a wooden box painted black internally to minimize any light reflections within the system.

\section{Results of the Spatial Response Testing}

For this report, we included the results for both sensors. The tabulated data results are summarized in Tables 2 through 5 and key spatial characteristics given in Figures 12,13, 14, and 15. Tables 2 through 5 list the results of the sensor response, both with the lens and with the lens removed. For each altitude angle, we averaged the angular response of the sensor over azimuth planes. We then calculated the normalized response by dividing the average of the sensor response by the average response at zero degree altitude angle. We also calculated the cosine response to demonstrate that the sensors have a much narrower cone of acceptance than a cosine response. Figures 12 though 15 show the normalized and cosine response for each sensor with and without lens.

Table 2-Spatial response of Sensor \#1 With Lens

\begin{tabular}{|c|l|l|l|l|l|l|l|l|l|l|l|}
\hline $\begin{array}{c}\text { Azimuth } \\
\rightarrow\end{array}$ & -90 & -80 & -70 & -60 & -50 & -40 & -30 & -20 & -10 & 0 & 10 \\
\hline Altitude & & & & & & & & & & & \\
\hline 0 & 8.30 & 8.30 & 8.30 & 8.30 & 8.30 & 8.30 & 8.30 & 8.30 & 8.30 & 8.30 & 8.30 \\
\hline 10 & 3.43 & 3.32 & 3.23 & 3.18 & 3.15 & 3.15 & 3.18 & 3.25 & 3.36 & 3.43 & 3.32 \\
\hline 20 & 1.36 & 1.32 & 1.28 & 1.25 & 1.21 & 1.19 & 1.17 & 1.17 & 1.18 & 1.36 & 1.32 \\
\hline 30 & 0.80 & 0.78 & 0.76 & 0.73 & 0.71 & 0.70 & 0.69 & 0.68 & 0.68 & 0.80 & 0.78 \\
\hline 40 & 0.47 & 0.46 & 0.45 & 0.44 & 0.43 & 0.43 & 0.43 & 0.43 & 0.43 & 0.47 & 0.46 \\
\hline 50 & 0.19 & 0.19 & 0.19 & 0.19 & 0.19 & 0.20 & 0.20 & 0.20 & 0.20 & 0.19 & 0.19 \\
\hline 60 & 0.10 & 0.10 & 0.10 & 0.10 & 0.10 & 0.10 & 0.11 & 0.11 & 0.11 & 0.10 & 0.10 \\
\hline 70 & 0.05 & 0.05 & 0.05 & 0.05 & 0.05 & 0.05 & 0.05 & 0.06 & 0.06 & 0.05 & 0.05 \\
\hline 80 & 0.03 & 0.03 & 0.03 & 0.03 & 0.03 & 0.03 & 0.03 & 0.03 & 0.03 & 0.03 & 0.03 \\
\hline 90 & 0.03 & 0.03 & 0.03 & 0.03 & 0.03 & 0.03 & 0.03 & 0.03 & 0.03 & 0.03 & 0.03 \\
\hline
\end{tabular}

Table 2- Continued

\begin{tabular}{|c|l|l|l|l|l|l|l|l|l|l|l|}
\hline $\begin{array}{c}\text { Azimuth } \\
\rightarrow\end{array}$ & 20 & 30 & 40 & 50 & 60 & 70 & 80 & 90 & 100 & 110 & 120 \\
\hline Altitude & & & & & & & & & & & \\
\hline 0 & 8.30 & 8.30 & 8.30 & 8.30 & 8.30 & 8.30 & 8.30 & 8.30 & 8.30 & 8.30 & 8.30 \\
\hline 10 & 3.99 & 4.27 & 4.63 & 5.06 & 5.61 & 6.27 & 7.03 & 8.30 & 8.30 & 8.30 & 8.30 \\
\hline 20 & 1.27 & 1.33 & 1.40 & 1.50 & 1.62 & 1.75 & 1.89 & 3.51 & 3.69 & 3.91 & 3.94 \\
\hline 30 & 0.70 & 0.72 & 0.75 & 0.79 & 0.83 & 0.89 & 0.93 & 1.29 & 1.33 & 1.37 & 1.40 \\
\hline 40 & 0.44 & 0.45 & 0.47 & 0.49 & 0.51 & 0.53 & 0.55 & 0.73 & 0.75 & 0.78 & 0.80 \\
\hline 50 & 0.21 & 0.22 & 0.22 & 0.23 & 0.24 & 0.24 & 0.25 & 0.41 & 0.42 & 0.44 & 0.45 \\
\hline 60 & 0.11 & 0.11 & 0.11 & 0.11 & 0.11 & 0.11 & 0.11 & 0.15 & 0.15 & 0.15 & 0.15 \\
\hline 70 & 0.06 & 0.06 & 0.06 & 0.06 & 0.06 & 0.06 & 0.06 & 0.08 & 0.08 & 0.07 & 0.07 \\
\hline 80 & 0.03 & 0.03 & 0.03 & 0.03 & 0.03 & 0.03 & 0.03 & 0.04 & 0.04 & 0.04 & 0.04 \\
\hline 90 & 0.03 & 0.03 & 0.03 & 0.03 & 0.03 & 0.03 & 0.03 & 0.03 & 0.03 & 0.03 & 0.03 \\
\hline
\end{tabular}


Table 2- Continued

\begin{tabular}{|c|l|l|l|l|l|l|l|l|l|l|l|}
\hline $\begin{array}{c}\text { Azimuth } \\
\rightarrow\end{array}$ & 130 & 140 & 150 & 160 & 170 & 180 & 190 & 200 & 210 & 220 & 230 \\
\hline Altitude & & & & & & & & & & & \\
\hline 0 & 8.30 & 8.30 & 8.30 & 8.30 & 8.30 & 8.30 & 8.30 & 8.30 & 8.30 & 8.30 & 8.30 \\
\hline 10 & 8.30 & 8.30 & 8.30 & 8.30 & 8.30 & 8.29 & 8.30 & 8.30 & 8.30 & 8.30 & 7.75 \\
\hline 20 & 3.99 & 3.88 & 3.79 & 3.57 & 3.40 & 3.15 & 2.94 & 2.70 & 2.54 & 2.37 & 2.24 \\
\hline 30 & 1.43 & 1.43 & 1.43 & 1.40 & 1.39 & 1.34 & 1.30 & 1.25 & 1.22 & 1.17 & 1.14 \\
\hline 40 & 0.82 & 0.83 & 0.83 & 0.83 & 0.83 & 0.82 & 0.80 & 0.77 & 0.75 & 0.73 & 0.71 \\
\hline 50 & 0.46 & 0.46 & 0.46 & 0.45 & 0.45 & 0.44 & 0.44 & 0.42 & 0.41 & 0.39 & 0.38 \\
\hline 60 & 0.14 & 0.14 & 0.14 & 0.14 & 0.13 & 0.13 & 0.13 & 0.13 & 0.13 & 0.12 & 0.12 \\
\hline 70 & 0.07 & 0.07 & 0.07 & 0.07 & 0.07 & 0.07 & 0.07 & 0.07 & 0.07 & 0.06 & 0.07 \\
\hline 80 & 0.04 & 0.04 & 0.04 & 0.04 & 0.04 & 0.04 & 0.04 & 0.04 & 0.04 & 0.04 & 0.04 \\
\hline 90 & 0.03 & 0.03 & 0.03 & 0.03 & 0.03 & 0.03 & 0.03 & 0.03 & 0.03 & 0.03 & 0.03 \\
\hline
\end{tabular}

Table 2- Continued

\begin{tabular}{|c|c|c|c|c|c|c|}
\hline $\begin{array}{l}\text { Azimuth } \\
\rightarrow\end{array}$ & 240 & 250 & 260 & \multirow{2}{*}{$\begin{array}{l}\text { Average } \\
\text { Response }\end{array}$} & \multirow{2}{*}{$\begin{array}{l}\text { Normalized } \\
\text { Response }\end{array}$} & \multirow[t]{2}{*}{$\begin{array}{l}\text { Cosine } \\
\text { Response }\end{array}$} \\
\hline Altitude & & & & & & \\
\hline 0 & 8.30 & 8.30 & 8.30 & 8.300 & 1.000 & 1.000 \\
\hline 10 & 7.19 & 6.73 & 6.38 & 6.045 & 0.728 & 0.985 \\
\hline 20 & 2.10 & 2.00 & 1.90 & 2.220 & 0.267 & 0.940 \\
\hline 30 & 1.09 & 1.05 & 1.00 & 1.015 & 0.122 & 0.866 \\
\hline 40 & 0.68 & 0.65 & 0.62 & 0.611 & 0.074 & 0.766 \\
\hline 50 & 0.35 & 0.34 & 0.32 & 0.313 & 0.038 & 0.643 \\
\hline 60 & 0.12 & 0.12 & 0.12 & 0.120 & 0.014 & 0.500 \\
\hline 70 & 0.07 & 0.07 & 0.07 & 0.063 & 0.008 & 0.342 \\
\hline 80 & 0.04 & 0.04 & 0.03 & 0.035 & 0.004 & 0.174 \\
\hline 90 & 0.03 & 0.03 & 0.03 & 0.030 & 0.004 & 0.000 \\
\hline
\end{tabular}


Table 3-Spatial response of Sensor \#1 Without Lens

\begin{tabular}{|c|l|l|l|l|l|l|l|l|l|l|l|}
\hline $\begin{array}{c}\text { Azimuth } \\
\rightarrow\end{array}$ & -90 & -80 & -70 & -60 & -50 & -40 & -30 & -20 & -10 & 0 & 10 \\
\hline Altitude & & & & & & & & & & & \\
\hline 0 & 4.64 & 4.62 & 4.61 & 5.47 & 5.49 & 5.49 & 5.50 & 5.47 & 5.44 & 5.51 & 5.53 \\
\hline 10 & 4.13 & 4.01 & 3.95 & 4.68 & 4.72 & 4.75 & 4.69 & 4.52 & 4.32 & 4.24 & 4.10 \\
\hline 20 & 3.32 & 3.42 & 3.56 & 4.44 & 4.31 & 4.22 & 4.00 & 3.72 & 3.47 & 3.41 & 3.45 \\
\hline 30 & 2.02 & 1.95 & 1.70 & 1.69 & 1.51 & 1.42 & 1.21 & 1.10 & 1.11 & 1.16 & 1.12 \\
\hline 40 & 0.75 & 0.75 & 0.72 & 0.82 & 0.74 & 0.70 & 0.68 & 0.70 & 0.76 & 0.81 & 0.79 \\
\hline 50 & 0.59 & 0.58 & 0.59 & 0.70 & 0.65 & 0.61 & 0.60 & 0.59 & 0.66 & 0.70 & 0.69 \\
\hline 60 & 0.52 & 0.51 & 0.52 & 0.60 & 0.59 & 0.52 & 0.49 & 0.50 & 0.57 & 0.61 & 0.60 \\
\hline 70 & 0.45 & 0.44 & 0.25 & 0.35 & 0.30 & 0.25 & 0.22 & 0.25 & 0.29 & 0.61 & 0.61 \\
\hline 80 & 0.16 & 0.22 & 0.24 & 0.21 & 0.21 & 0.15 & 0.13 & 0.15 & 0.15 & 0.17 & 0.19 \\
\hline 90 & 0.08 & 0.06 & 0.07 & 0.08 & 0.20 & 0.07 & 0.12 & 0.18 & 0.22 & 0.19 & 0.32 \\
\hline
\end{tabular}

Table 3- Continued

\begin{tabular}{|c|l|l|l|l|l|l|l|l|l|l|l|}
\hline $\begin{array}{c}\text { Azimuth } \\
\rightarrow\end{array}$ & 20 & 30 & 40 & 50 & 60 & 70 & 80 & 90 & 100 & 110 & 120 \\
\hline Altitude & & & & & & & & & & & \\
\hline 0 & 5.54 & 5.57 & 5.61 & 5.66 & 5.72 & 5.79 & 5.87 & 4.64 & 4.62 & 4.61 & 5.47 \\
\hline 10 & 4.01 & 4.12 & 4.34 & 4.51 & 4.56 & 4.74 & 4.88 & 4.43 & 4.49 & 5.48 & 5.86 \\
\hline 20 & 3.34 & 3.39 & 3.51 & 3.86 & 4.17 & 4.59 & 4.97 & 5.23 & 5.05 & 5.90 & 6.06 \\
\hline 30 & 1.12 & 1.14 & 1.15 & 1.15 & 1.16 & 1.22 & 1.31 & 2.34 & 2.62 & 3.62 & 3.98 \\
\hline 40 & 0.78 & 0.77 & 0.78 & 0.78 & 0.80 & 0.83 & 0.90 & 0.86 & 0.94 & 1.18 & 1.22 \\
\hline 50 & 0.67 & 0.65 & 0.64 & 0.64 & 0.64 & 0.66 & 0.69 & 0.63 & 0.64 & 0.75 & 0.81 \\
\hline 60 & 0.59 & 0.60 & 0.57 & 0.56 & 0.54 & 0.56 & 0.57 & 0.49 & 0.48 & 0.61 & 0.69 \\
\hline 70 & 0.62 & 0.60 & 0.58 & 0.55 & 0.49 & 0.50 & 0.54 & 0.43 & 0.43 & 0.55 & 0.14 \\
\hline 80 & 0.46 & 0.53 & 0.46 & 0.24 & 0.30 & 0.47 & 0.44 & 0.37 & 0.36 & 0.37 & 0.29 \\
\hline 90 & 0.31 & 0.41 & 0.16 & 0.23 & 0.31 & 0.10 & 0.11 & 0.27 & 0.11 & 0.33 & 0.33 \\
\hline
\end{tabular}

Table 3- Continued

\begin{tabular}{|c|c|c|c|c|c|c|c|c|c|c|c|}
\hline $\begin{array}{c}\text { Azimuth } \\
\rightarrow\end{array}$ & 130 & 140 & 150 & 160 & 170 & 180 & 190 & 200 & 210 & 220 & 230 \\
\hline Altitude & & & & & & & & & & & \\
\hline 0 & 5.49 & 5.49 & 5.50 & 5.47 & 5.44 & 5.51 & 5.53 & 5.54 & 5.57 & 5.61 & 5.66 \\
\hline 10 & 6.22 & 6.38 & 6.35 & 6.16 & 6.03 & 5.95 & 5.81 & 5.74 & 5.74 & 5.77 & 5.66 \\
\hline 20 & 6.40 & 6.75 & 7.03 & 7.00 & 6.88 & 6.58 & 5.94 & 5.55 & 5.19 & 4.99 & 4.80 \\
\hline 30 & 4.56 & 5.08 & 5.54 & 5.65 & 5.83 & 6.01 & 5.76 & 5.47 & 5.60 & 5.77 & 5.76 \\
\hline 40 & 1.36 & 1.48 & 1.61 & 1.68 & 1.60 & 1.55 & 1.38 & 1.10 & 1.01 & 1.36 & 1.55 \\
\hline 50 & 0.86 & 1.06 & 1.06 & 0.94 & 0.77 & 0.62 & 0.68 & 0.75 & 0.77 & 0.77 & 0.73 \\
\hline 60 & 0.71 & 0.84 & 0.92 & 0.75 & 0.61 & 0.53 & 0.65 & 0.67 & 0.62 & 0.59 & 0.58 \\
\hline 70 & 0.13 & 0.40 & 0.14 & 0.27 & 0.50 & 0.53 & 0.56 & 0.54 & 0.52 & 0.51 & 0.49 \\
\hline 80 & 0.19 & 0.14 & 0.10 & 0.15 & 0.48 & 0.60 & 0.55 & 0.52 & 0.54 & 0.49 & 0.30 \\
\hline 90 & 0.22 & 0.07 & 0.06 & 0.11 & 0.46 & 0.16 & 0.08 & 0.07 & 0.35 & 0.12 & 0.08 \\
\hline
\end{tabular}


Table 3- Continued

\begin{tabular}{|c|c|c|c|c|c|c|}
\hline $\begin{array}{c}\text { Azimuth } \\
\rightarrow\end{array}$ & 240 & 250 & 260 & $\begin{array}{l}\text { Average } \\
\text { Response }\end{array}$ & $\begin{array}{l}\text { Normalized } \\
\text { Response }\end{array}$ & $\begin{array}{l}\text { Cosine } \\
\text { Response }\end{array}$ \\
\hline Altitude & & & & 5.718 & 1.000 & 1.000 \\
\hline 0 & 5.72 & 5.79 & 5.87 & 5.418 & 0.927 & 0.985 \\
\hline 10 & 5.39 & 5.15 & 5.03 & 5.025 & 0.877 & 0.940 \\
\hline 20 & 4.48 & 4.11 & 3.93 & 4.751 & 0.570 & 0.866 \\
\hline 30 & 5.12 & 4.40 & 3.80 & 3.088 & 0.192 & 0.766 \\
\hline 40 & 1.38 & 1.25 & 1.17 & 1.043 & 0.131 & 0.643 \\
\hline 50 & 0.70 & 0.69 & 0.72 & 0.708 & 0.13 & 0.500 \\
\hline 60 & 0.57 & 0.59 & 0.60 & 0.598 & 0.110 & 0.342 \\
\hline 70 & 0.49 & 0.52 & 0.53 & 0.433 & 0.080 & 0.174 \\
\hline 80 & 0.18 & 0.17 & 0.19 & 0.302 & 0.056 & 0.000 \\
\hline 90 & 0.07 & 0.11 & 0.11 & 0.176 & 0.032 & 0.00 \\
\hline
\end{tabular}


Table 4-Spatial response of Sensor \#2 With Lens

\begin{tabular}{|c|l|l|l|l|l|l|l|l|l|l|l|}
\hline $\begin{array}{c}\text { Azimuth } \\
\rightarrow\end{array}$ & -90 & -80 & -70 & -60 & -50 & -40 & -30 & -20 & -10 & 0 & 10 \\
\hline Altitude & & & & & & & & & & & \\
\hline 0 & 4.75 & 4.70 & 4.68 & 4.68 & 4.69 & 4.72 & 4.77 & 4.84 & 4.93 & 5.03 & 5.15 \\
\hline 10 & 1.51 & 1.52 & 1.53 & 1.54 & 1.57 & 1.62 & 1.67 & 1.73 & 1.80 & 1.86 & 1.94 \\
\hline 20 & 0.57 & 0.57 & 0.58 & 0.58 & 0.59 & 0.60 & 0.60 & 0.61 & 0.61 & 0.62 & 0.63 \\
\hline 30 & 0.34 & 0.33 & 0.34 & 0.34 & 0.34 & 0.35 & 0.35 & 0.35 & 0.35 & 0.35 & 0.35 \\
\hline 40 & 0.23 & 0.24 & 0.24 & 0.24 & 0.24 & 0.24 & 0.24 & 0.25 & 0.25 & 0.25 & 0.25 \\
\hline 50 & 0.16 & 0.16 & 0.16 & 0.16 & 0.16 & 0.16 & 0.17 & 0.17 & 0.17 & 0.17 & 0.17 \\
\hline 60 & 0.09 & 0.09 & 0.09 & 0.09 & 0.09 & 0.10 & 0.10 & 0.10 & 0.10 & 0.10 & 0.10 \\
\hline 70 & 0.07 & 0.07 & 0.07 & 0.07 & 0.08 & 0.08 & 0.08 & 0.08 & 0.08 & 0.08 & 0.07 \\
\hline 80 & 0.06 & 0.06 & 0.06 & 0.06 & 0.06 & 0.06 & 0.06 & 0.06 & 0.06 & 0.06 & 0.06 \\
\hline 90 & 0.06 & 0.06 & 0.06 & 0.06 & 0.06 & 0.06 & 0.06 & 0.06 & 0.06 & 0.06 & 0.06 \\
\hline
\end{tabular}

Table 4- Continued

\begin{tabular}{|c|l|l|l|l|l|l|l|l|l|l|l|}
\hline $\begin{array}{c}\text { Azimuth } \\
\rightarrow\end{array}$ & 20 & 30 & 40 & 50 & 60 & 70 & 80 & 90 & 100 & 110 & 120 \\
\hline Altitude & & & & & & & & & & & \\
\hline 0 & 5.28 & 5.41 & 5.55 & 5.69 & 5.82 & 5.94 & 6.04 & 4.75 & 4.70 & 4.68 & 4.68 \\
\hline 10 & 2.03 & 2.14 & 2.28 & 2.45 & 2.63 & 2.81 & 2.96 & 4.92 & 4.96 & 4.94 & 4.85 \\
\hline 20 & 0.65 & 0.67 & 0.70 & 0.73 & 0.77 & 0.81 & 0.84 & 1.42 & 1.41 & 1.41 & 1.37 \\
\hline 30 & 0.35 & 0.35 & 0.36 & 0.37 & 0.39 & 0.39 & 0.40 & 0.51 & 0.50 & 0.51 & 0.50 \\
\hline 40 & 0.25 & 0.25 & 0.26 & 0.26 & 0.27 & 0.27 & 0.27 & 0.30 & 0.30 & 0.30 & 0.30 \\
\hline 50 & 0.18 & 0.18 & 0.18 & 0.18 & 0.18 & 0.18 & 0.18 & 0.20 & 0.20 & 0.20 & 0.20 \\
\hline 60 & 0.10 & 0.10 & 0.10 & 0.09 & 0.09 & 0.09 & 0.09 & 0.10 & 0.09 & 0.09 & 0.09 \\
\hline 70 & 0.07 & 0.07 & 0.07 & 0.07 & 0.07 & 0.07 & 0.07 & 0.07 & 0.07 & 0.07 & 0.07 \\
\hline 80 & 0.06 & 0.06 & 0.06 & 0.06 & 0.06 & 0.06 & 0.06 & 0.06 & 0.06 & 0.06 & 0.06 \\
\hline 90 & 0.06 & 0.06 & 0.06 & 0.06 & 0.06 & 0.06 & 0.06 & 0.06 & 0.06 & 0.06 & 0.06 \\
\hline
\end{tabular}

Table 4- Continued

\begin{tabular}{|c|l|l|l|l|l|l|l|l|l|l|l|}
\hline $\begin{array}{c}\text { Azimuth } \\
\rightarrow\end{array}$ & 130 & 140 & 150 & 160 & 170 & 180 & 190 & 200 & 210 & 220 & 230 \\
\hline Altitude & & & & & & & & & & & \\
\hline 0 & 4.69 & 4.72 & 4.77 & 4.84 & 4.93 & 5.03 & 5.15 & 5.28 & 5.41 & 5.55 & 5.69 \\
\hline 10 & 4.70 & 4.49 & 4.24 & 3.97 & 3.70 & 3.45 & 3.22 & 3.03 & 2.88 & 2.76 & 2.67 \\
\hline 20 & 1.34 & 1.28 & 1.23 & 1.16 & 1.10 & 1.03 & 0.97 & 0.92 & 0.89 & 0.85 & 0.84 \\
\hline 30 & 0.50 & 0.50 & 0.50 & 0.48 & 0.47 & 0.45 & 0.44 & 0.43 & 0.42 & 0.41 & 0.40 \\
\hline 40 & 0.30 & 0.31 & 0.31 & 0.31 & 0.30 & 0.29 & 0.29 & 0.28 & 0.28 & 0.27 & 0.27 \\
\hline 50 & 0.19 & 0.19 & 0.19 & 0.19 & 0.19 & 0.19 & 0.19 & 0.18 & 0.18 & 0.18 & 0.18 \\
\hline 60 & 0.09 & 0.09 & 0.09 & 0.09 & 0.09 & 0.09 & 0.09 & 0.09 & 0.09 & 0.09 & 0.09 \\
\hline 70 & 0.07 & 0.07 & 0.07 & 0.07 & 0.07 & 0.07 & 0.07 & 0.07 & 0.07 & 0.07 & 0.07 \\
\hline 80 & 0.06 & 0.06 & 0.06 & 0.06 & 0.06 & 0.06 & 0.06 & 0.06 & 0.06 & 0.06 & 0.06 \\
\hline 90 & 0.06 & 0.06 & 0.06 & 0.06 & 0.06 & 0.06 & 0.06 & 0.06 & 0.06 & 0.06 & 0.06 \\
\hline
\end{tabular}


Table 4- Continued

\begin{tabular}{|c|l|l|r|r|r|r|}
\hline $\begin{array}{c}\text { Azimuth } \\
\rightarrow\end{array}$ & 240 & 250 & 260 & $\begin{array}{l}\text { Average } \\
\text { Response }\end{array}$ & $\begin{array}{r}\text { Normalized } \\
\text { Response }\end{array}$ & $\begin{array}{l}\text { Cosine } \\
\text { Response }\end{array}$ \\
\cline { 1 - 3 } Altitude & & & & 5.148 & 1.000 & 1.000 \\
\hline 0 & 5.82 & 5.94 & 6.04 & 2.836 & 0.551 & 0.985 \\
\hline 10 & 2.61 & 2.57 & 2.55 & 2.83 & 0.169 & 0.940 \\
\hline 20 & 0.82 & 0.82 & 0.81 & 0.872 & 0.079 & 0.866 \\
\hline 30 & 0.40 & 0.39 & 0.39 & 0.406 & 0.052 & 0.766 \\
\hline 40 & 0.26 & 0.26 & 0.26 & 0.269 & 0.035 & 0.643 \\
\hline 50 & 0.18 & 0.18 & 0.18 & 0.179 & 0.018 & 0.500 \\
\hline 60 & 0.09 & 0.09 & 0.10 & 0.093 & 0.014 & 0.342 \\
\hline 70 & 0.07 & 0.07 & 0.07 & 0.072 & 0.012 & 0.174 \\
\hline 80 & 0.06 & 0.06 & 0.06 & 0.060 & 0.060 \\
\hline 90 & 0.06 & 0.06 & 0.06 & 0.060 & 0.012 & 0.000 \\
\hline
\end{tabular}


Table 5-Spatial response of Sensor \#2 Without Lens

\begin{tabular}{|c|l|l|l|l|l|l|l|l|l|l|l|}
\hline $\begin{array}{c}\text { Azimuth } \\
\rightarrow\end{array}$ & -90 & -80 & -70 & -60 & -50 & -40 & -30 & -20 & -10 & 0 & 10 \\
\hline Altitude & & & & & & & & & & & \\
\hline 0 & 1.82 & 1.83 & 1.86 & 1.89 & 1.91 & 1.93 & 1.93 & 1.94 & 1.93 & 1.93 & 1.92 \\
\hline 10 & 1.93 & 1.90 & 1.87 & 1.86 & 1.86 & 1.89 & 1.91 & 1.94 & 1.93 & 1.91 & 1.96 \\
\hline 20 & 1.41 & 1.37 & 1.29 & 1.27 & 1.31 & 1.33 & 1.29 & 1.29 & 1.33 & 1.37 & 1.42 \\
\hline 30 & 0.59 & 0.65 & 0.63 & 0.59 & 0.57 & 0.55 & 0.51 & 0.52 & 0.50 & 0.50 & 0.44 \\
\hline 40 & 0.30 & 0.32 & 0.32 & 0.32 & 0.33 & 0.33 & 0.32 & 0.33 & 0.33 & 0.34 & 0.34 \\
\hline 50 & 0.31 & 0.31 & 0.31 & 0.31 & 0.30 & 0.30 & 0.30 & 0.29 & 0.29 & 0.29 & 0.29 \\
\hline 60 & 0.29 & 0.30 & 0.30 & 0.34 & 0.28 & 0.28 & 0.27 & 0.27 & 0.26 & 0.27 & 0.27 \\
\hline 70 & 0.28 & 0.27 & 0.28 & 0.30 & 0.27 & 0.27 & 0.26 & 0.26 & 0.25 & 0.26 & 0.26 \\
\hline 80 & 0.28 & 0.28 & 0.27 & 0.27 & 0.26 & 0.27 & 0.26 & 0.26 & 0.27 & 0.28 & 0.27 \\
\hline 90 & 0.25 & 0.25 & 0.23 & 0.23 & 0.15 & 0.12 & 0.14 & 0.12 & 0.11 & 0.25 & 0.23 \\
\hline
\end{tabular}

Table 5- Continued

\begin{tabular}{|c|l|l|l|l|l|l|l|l|l|l|l|}
\hline $\begin{array}{c}\text { Azimuth } \\
\rightarrow\end{array}$ & 20 & 30 & 40 & 50 & 60 & 70 & 80 & 90 & 100 & 110 & 120 \\
\hline Altitude & & & & & & & & & & & \\
\hline 0 & 1.92 & 1.91 & 1.92 & 1.93 & 1.94 & 1.96 & 1.98 & 1.82 & 1.83 & 1.86 & 1.89 \\
\hline 10 & 1.96 & 1.93 & 1.96 & 2.06 & 2.08 & 2.13 & 2.19 & 1.87 & 1.90 & 1.93 & 1.93 \\
\hline 20 & 1.57 & 1.66 & 1.87 & 1.96 & 1.97 & 2.08 & 2.14 & 2.36 & 2.38 & 2.41 & 2.51 \\
\hline 30 & 0.42 & 0.40 & 0.45 & 0.52 & 0.58 & 0.61 & 0.65 & 1.08 & 1.01 & 1.05 & 1.10 \\
\hline 40 & 0.31 & 0.29 & 0.31 & 0.33 & 0.33 & 0.35 & 0.36 & 0.39 & 0.39 & 0.38 & 0.37 \\
\hline 50 & 0.27 & 0.26 & 0.26 & 0.27 & 0.28 & 0.28 & 0.29 & 0.26 & 0.26 & 0.23 & 0.23 \\
\hline 60 & 0.25 & 0.24 & 0.24 & 0.25 & 0.26 & 0.27 & 0.27 & 0.24 & 0.23 & 0.21 & 0.20 \\
\hline 70 & 0.24 & 0.22 & 0.23 & 0.25 & 0.26 & 0.27 & 0.28 & 0.22 & 0.22 & 0.21 & 0.19 \\
\hline 80 & 0.24 & 0.22 & 0.22 & 0.24 & 0.24 & 0.24 & 0.22 & 0.22 & 0.20 & 0.15 & 0.12 \\
\hline 90 & 0.11 & 0.11 & 0.10 & 0.21 & 0.12 & 0.10 & 0.11 & 0.10 & 0.09 & 0.09 & 0.10 \\
\hline
\end{tabular}

Table 5- Continued

\begin{tabular}{|c|l|l|l|l|l|l|l|l|l|l|l|}
\hline $\begin{array}{c}\text { Azimuth } \\
\rightarrow\end{array}$ & 130 & 140 & 150 & 160 & 170 & 180 & 190 & 200 & 210 & 220 & 230 \\
\hline Altitude & & & & & & & & & & & \\
\hline 0 & 1.91 & 1.93 & 1.93 & 1.94 & 1.93 & 1.93 & 1.92 & 1.92 & 1.91 & 1.92 & 1.93 \\
\hline 10 & 1.94 & 1.97 & 1.96 & 1.95 & 1.91 & 1.88 & 1.83 & 1.79 & 1.79 & 1.81 & 1.81 \\
\hline 20 & 2.56 & 2.56 & 2.49 & 2.41 & 2.26 & 2.09 & 1.98 & 1.88 & 1.78 & 1.70 & 1.62 \\
\hline 30 & 1.21 & 1.36 & 1.60 & 1.58 & 1.43 & 1.30 & 1.30 & 1.29 & 1.31 & 1.19 & 0.99 \\
\hline 40 & 0.39 & 0.42 & 0.46 & 0.47 & 0.45 & 0.42 & 0.40 & 0.39 & 0.37 & 0.36 & 0.33 \\
\hline 50 & 0.25 & 0.27 & 0.30 & 0.31 & 0.29 & 0.27 & 0.27 & 0.27 & 0.26 & 0.26 & 0.27 \\
\hline 60 & 0.21 & 0.22 & 0.21 & 0.21 & 0.25 & 0.25 & 0.24 & 0.24 & 0.23 & 0.23 & 0.25 \\
\hline 70 & 0.21 & 0.22 & 0.24 & 0.21 & 0.25 & 0.24 & 0.22 & 0.22 & 0.21 & 0.22 & 0.23 \\
\hline 80 & 0.16 & 0.19 & 0.21 & 0.21 & 0.25 & 0.24 & 0.22 & 0.22 & 0.23 & 0.24 & 0.26 \\
\hline 90 & 0.10 & 0.12 & 0.11 & 0.12 & 0.24 & 0.18 & 0.10 & 0.10 & 0.16 & 0.25 & 0.24 \\
\hline
\end{tabular}


Table 5- Continued

\begin{tabular}{|c|l|l|r|r|r|r|}
\hline $\begin{array}{c}\text { Azimuth } \\
\rightarrow\end{array}$ & 240 & 250 & 260 & $\begin{array}{l}\text { Average } \\
\text { Response }\end{array}$ & $\begin{array}{r}\text { Normalized } \\
\text { Response }\end{array}$ & $\begin{array}{l}\text { Cosine } \\
\text { Response }\end{array}$ \\
\cline { 1 - 3 } Altitude & & & & 1.914 & 1.000 & 1.000 \\
\hline 0 & 1.94 & 1.96 & 1.98 & 1.914 & 1.000 & 0.985 \\
\hline 10 & 1.79 & 1.77 & 1.80 & 1.914 & 0.953 & 0.940 \\
\hline 20 & 1.58 & 1.56 & 1.59 & 1.824 & 0.451 & 0.866 \\
\hline 30 & 0.81 & 0.86 & 0.92 & 0.863 & 0.185 & 0.766 \\
\hline 40 & 0.30 & 0.28 & 0.29 & 0.353 & 0.146 & 0.643 \\
\hline 50 & 0.27 & 0.27 & 0.29 & 0.279 & 0.133 & 0.500 \\
\hline 60 & 0.26 & 0.27 & 0.27 & 0.254 & 0.127 & 0.342 \\
\hline 70 & 0.25 & 0.25 & 0.25 & 0.244 & 0.123 & 0.174 \\
\hline 80 & 0.26 & 0.25 & 0.25 & 0.235 & 0.081 & 0.000 \\
\hline 90 & 0.11 & 0.14 & 0.26 & 0.154 & 0.000 \\
\hline
\end{tabular}


Sensor

Altitude Measurement shaft

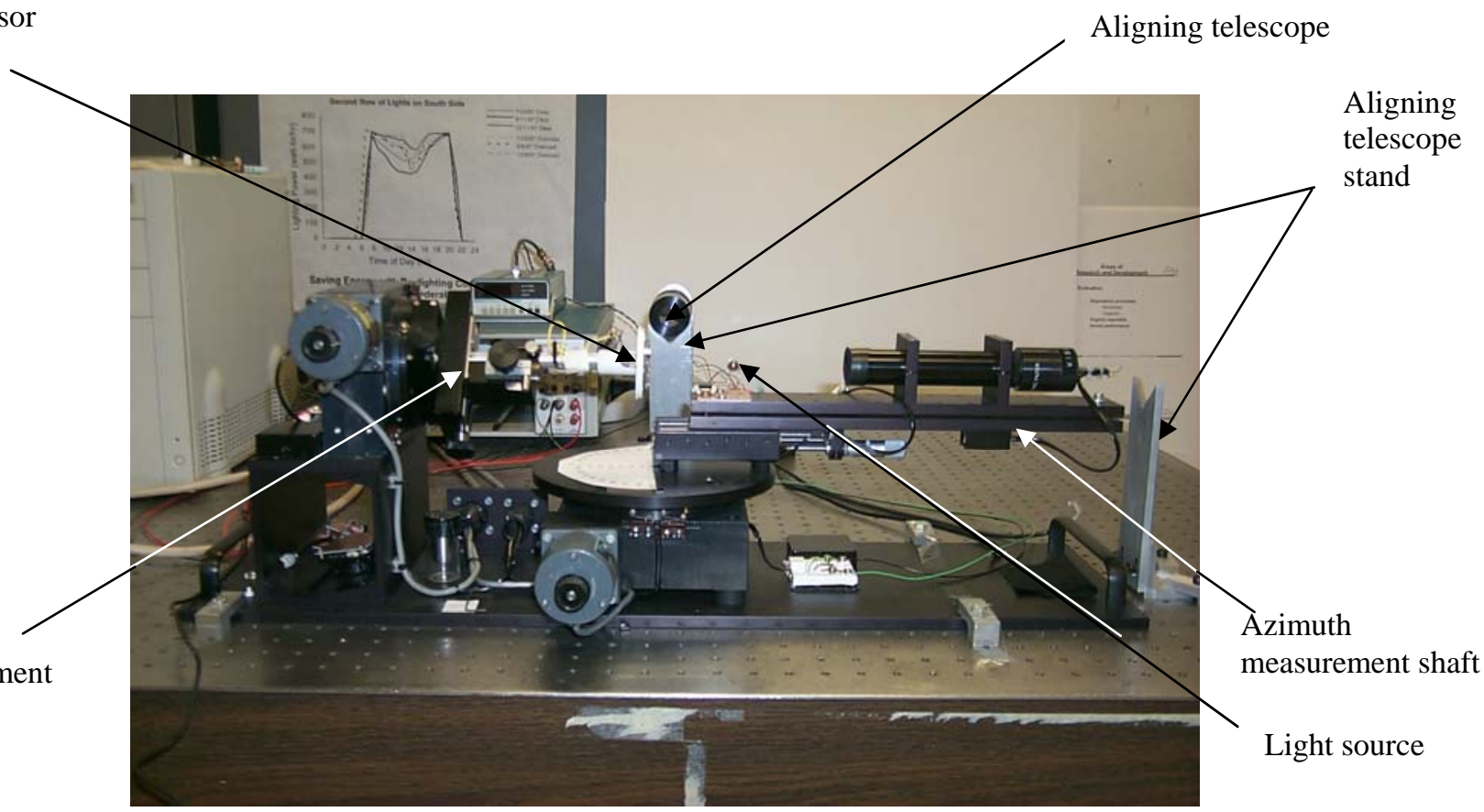

Figure 9- Goniometer used in spatial response measurement

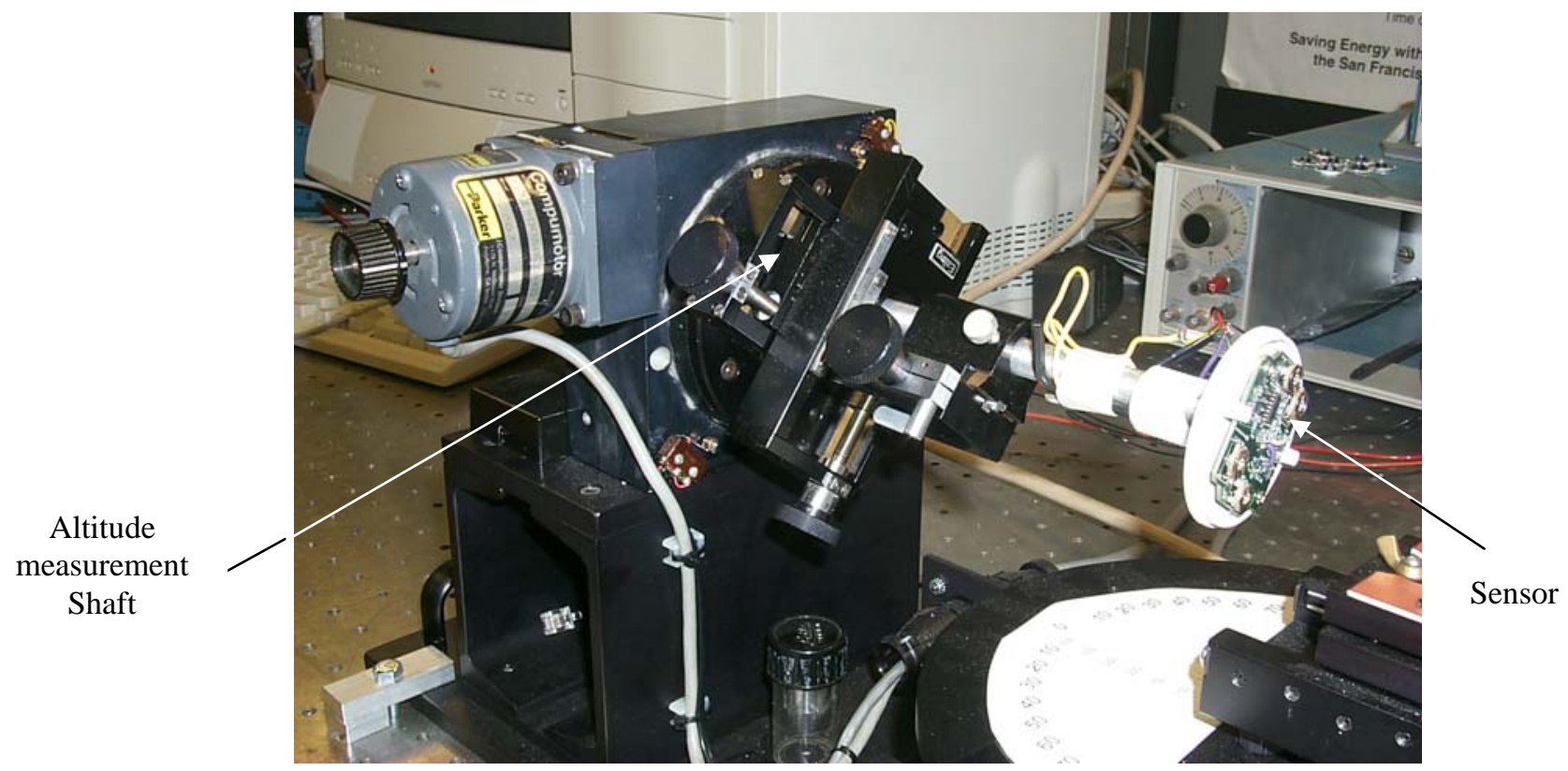

Figure 10- Position of sensor in spatial measurement (shown with sensor's front cover removed) 


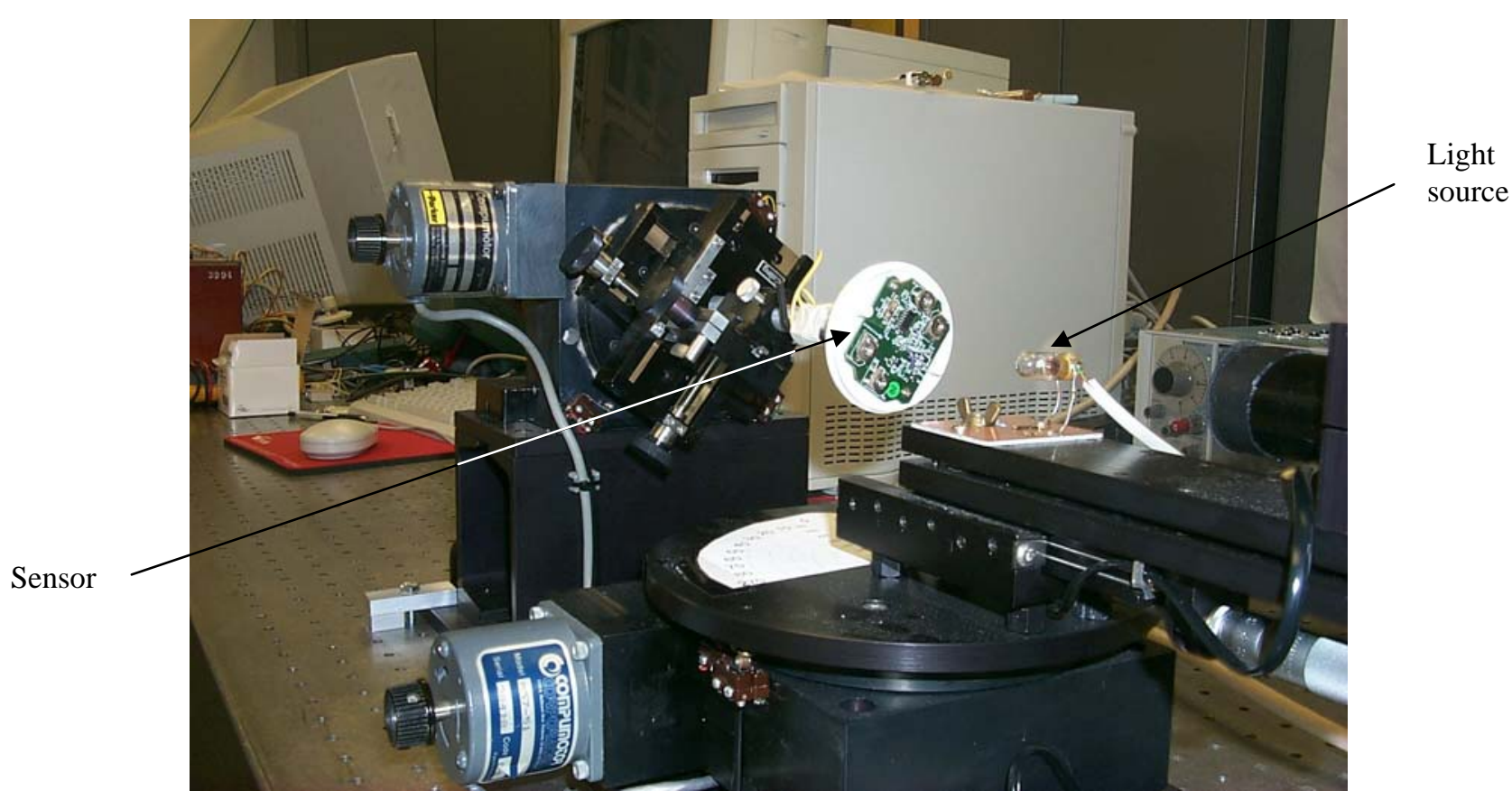

Figure 11- Sensor and light source position in spatial measurements (shown with the front cover of the sensor removed.) 
Figure 12-Spatial Response for Sensor \#1 with Lens

Response Averaged Over All Azimuth Planes

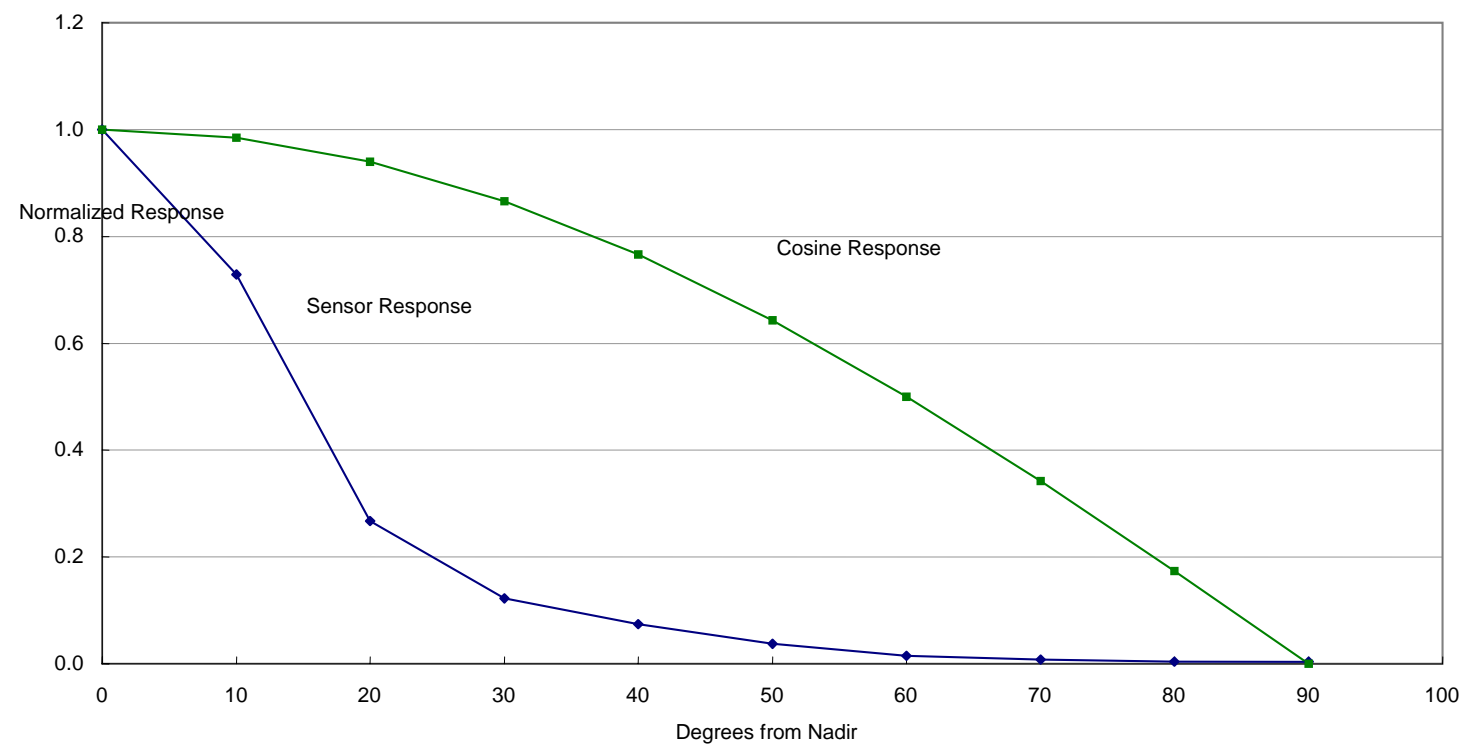

Figure 13-Spatial Response for Sensor \#1 without Lens Response Averaged Over All Azimuth Planes

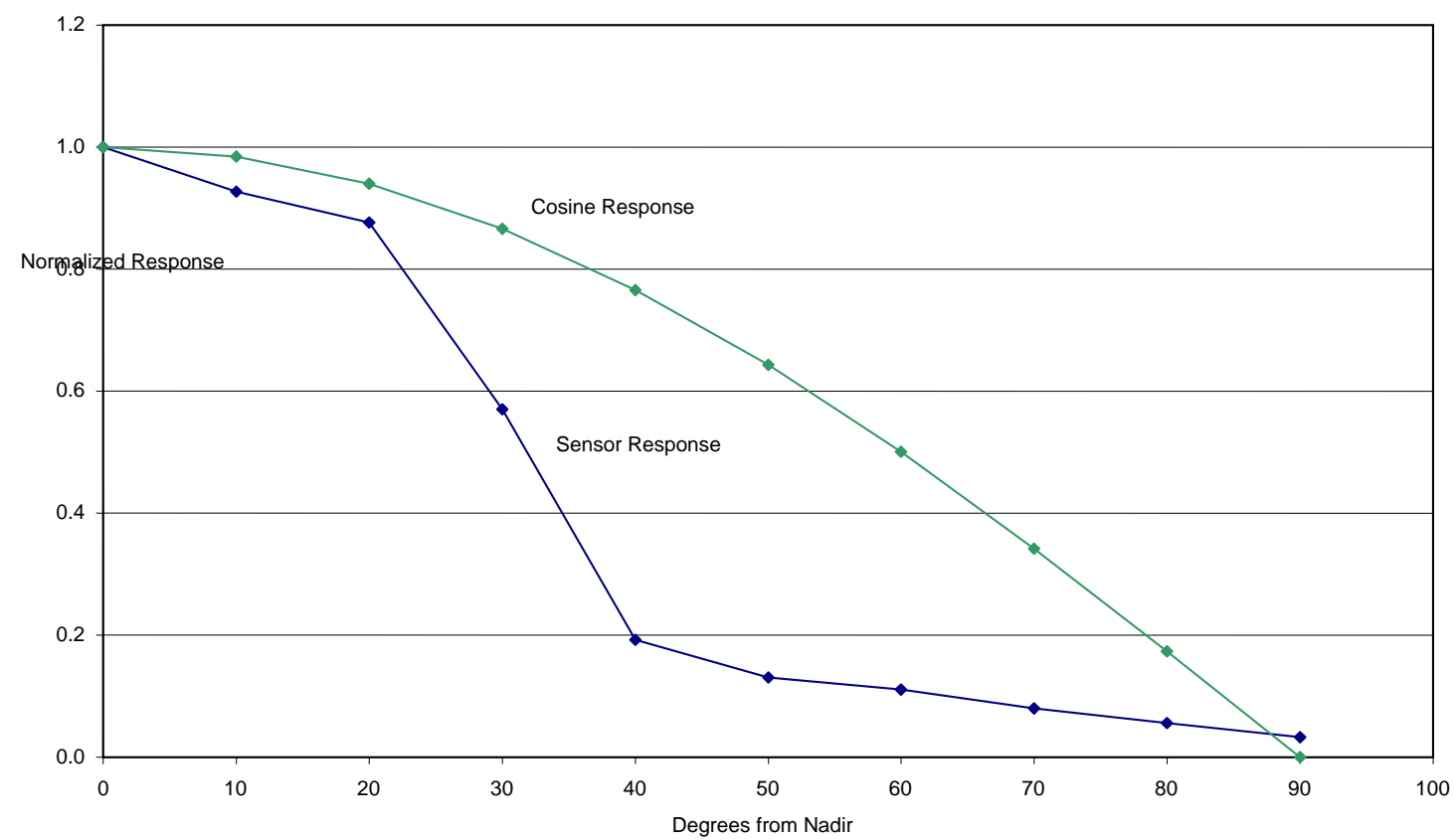


Figure 14-Spatial Response for Sensor \#2 with Lens Response Averaged Over All Azimuth Planes

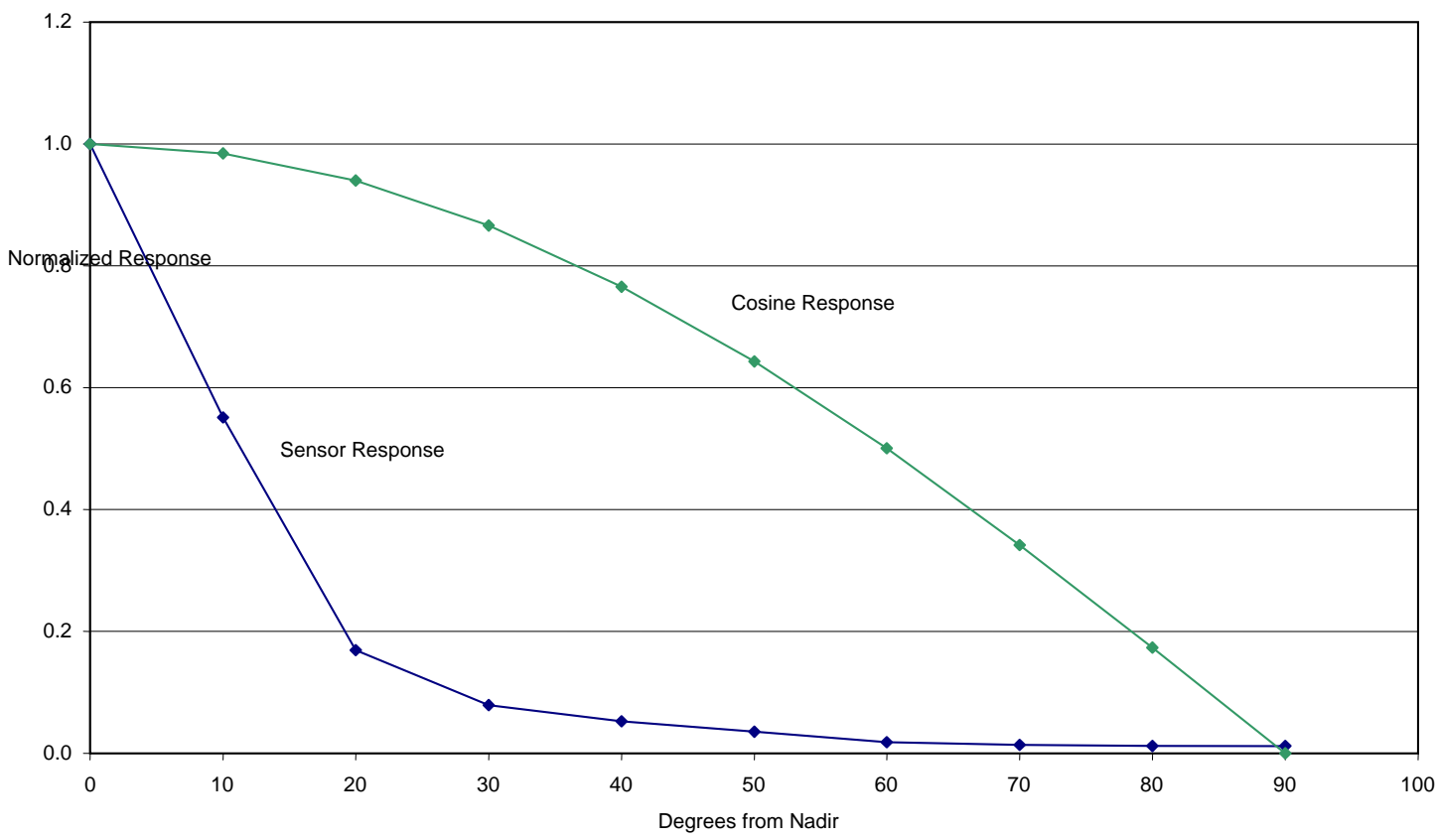

Figure 15-Spatial Response for Sensor \#2 without Lens

Response Averaged Over All Azimuth Planes

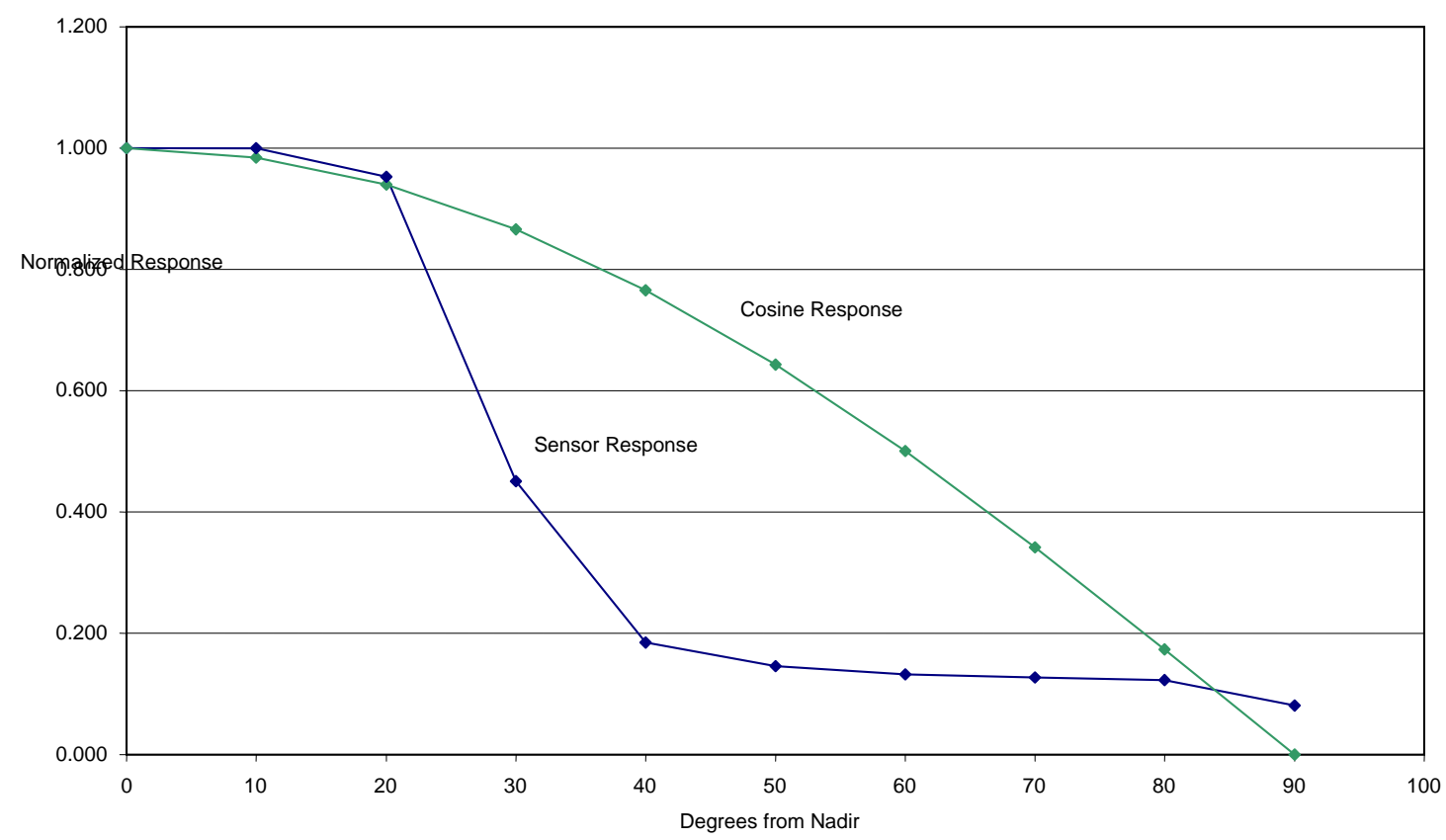


Figure 16. Polar Plot of Spatial Responsivity Function of Sensor \#1

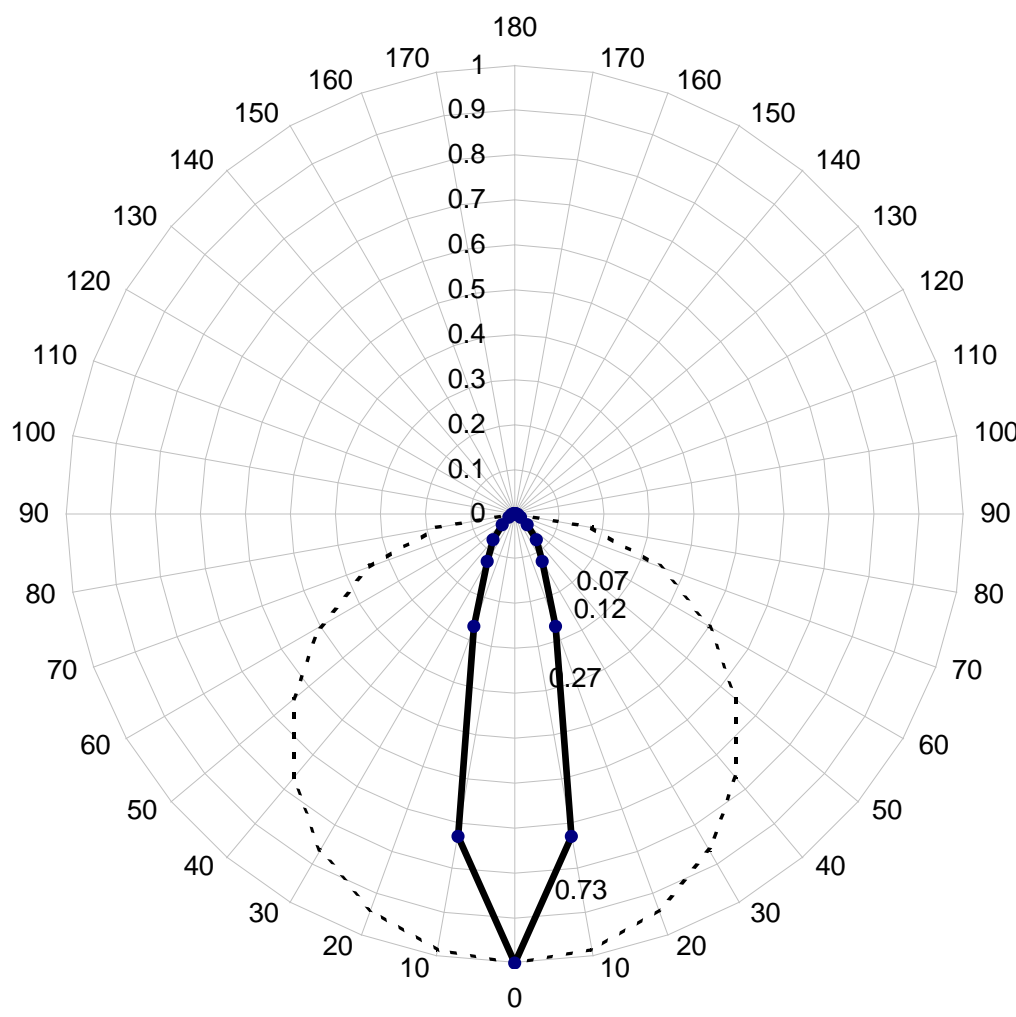




\section{Recommendations}

The spectral response function of the LS-201 photosensor is likely to be acceptable in many daylighting applications. Although its response curve is slightly narrower than the photopic curve, and the "peak" shifted slightly to the blue, it is better color-corrected than many of the photosensors currently on the market. One particularly favorable attribute is its insensivity to infrared radiation. Most photosensors available today for lighting control applications are based on silicon photodiodes which, if uncorrected, are more sensitive to the infrared portion of the electromagnetic spectrum than to visible light. Previous work has shown that photosensor sensitivity to IR can cause significant errors in control systems.

Because of the different physical principle underlying the operation of the LS-201, the sensor is entirely blind to IR. Essentially, photons with wavelengths greater than about $560 \mathrm{~nm}$ (for this LED) have insufficient energy to excite any charge carriers within the LED. Conversely, photons with shorter wavelengths than the peak response of the LED can excite charge carriers within the LED and this action tends to shift the "peak" of the spectral response function about $40 \mathrm{~nm}$ towards the blue. As a result, the sensor serves (fortuitously) as a decent scotopic meter. This has intriguing implications for the "Clear Vision” project that PG\&E is investigating (see Emerging Technologies Coordinating Council database). Since a daylight control system built around the LS-201 would be more sensitive to the scotopic function than the photopic, the energy savings benefits of the scotopic research could be directly realized with an automatic control system assembled from hardware available today.

The spatial response function of the LS-201 as it currently stands is probably adequate for many applications. However, its narrow field of view (FOV), effectively a cone of 15 degree half-angle, may be overly sensitive to changes in the luminances in the FOV resulting in unexpected behavior of the lighting system under extreme conditions. Since the FOV for the sensor with the lens removed is significantly wider (a cone of 35 degree half-angle), it is the action of the lens itself that is causing the narrowing of the FOV. We would suggest replacing with the Fresnel lens with a slightly diffuse flat lens. This would judiciously broaden the FOV without compromising external appearance. A photosensor with a FOV of 35 degrees would not be sensitive to direct window light (which previous work indicates should be avoided) unless it were mounted very close to the window wall.

Overall, the LS-201 photosensor is the first commercially available control photosensor that we have seen that basically "gets it right." It implements the most appropriate control algorithm for daylighting ("sliding setpoint") as well as providing critical adjustments of gain, offset, and minimum trim. One cautionary note is that we have not run the simulations to ascertain that the correct range of commissioning adjustments have been realized in this product for daylit classroom applications. Furthermore, although all the correct commissioning adjustments are physically present, the sensor is a daunting array of trim pots and jumpers that can only be adjusted in the field by a person on a ladder. As it stands, the product could probably not pass the 5-minute commissioning test. However, it has been used successfully in recent building projects and as long as sufficient time is 
allowed in the building contract for commissioning by a qualified commissioning agent, the LS-201 may be an appropriate solution for many commercial daylighting applications.

\section{Ideas for Future Development}

With regards to product enhancement, it seems all the necessary adjustments have been realized in the physical circuit design using trim pots. Consequently, it should be straightforward to replace the trim pots with digital components (specifically 1-Wire digital potentiometers) of equivalent functionality. The use of 1-Wire devices in the photosensor provides network connectivity and allows the use of the 1-Wire communications protocol. This would allow the realization of a digitally-addressable photosensor that could be commissioned in the field remotely using a portable personal computer (PC) or even a PDA (i.e., PalmPilot). In order that such a sensor remain effective in the legacy analog world as well as the digital realm, we recommend that such a hybrid sensor be based on the IEEE P1451 Standard for Sensors and Actuators. The IEEE P1451 Standard provides a common format for digitally storing the sensor specifications (called the TEDS table) onboard the sensor itself. In addition, the Standard specifies the communications protocol for digitally transmitting sensor data onto a lowcost wired network. A sensor built to the IEEE P1451 Standard can continue to operate and add value to legacy analog equipment and still be ready for the emerging digitallyaddressable lighting market. 